\title{
Carbon and oxygen abundances in stellar populations ${ }^{\star}, \star \star$
}

\author{
P. E. Nissen ${ }^{1,2}$, Y. Q. Chen ${ }^{1}$, L. Carigi ${ }^{3}$, W. J. Schuster ${ }^{4}$, and G. Zhao ${ }^{1}$ \\ ${ }^{1}$ Key Laboratory of Optical Astronomy, National Astronomical Observatories, Chinese Academy of Sciences, 100012 Beijing, \\ PR China \\ 2 Stellar Astrophysics Centre, Department of Physics and Astronomy, Aarhus University, Ny Munkegade 120, 8000 Aarhus C, \\ Denmark \\ e-mail: pen@phys.au.dk \\ 3 Instituto de Astronomía, Universidad Nacional Autónoma de México, AP 70-264, 04510 México DF, Mexico \\ 4 Observatorio Astronómico Nacional, Universidad Nacional Autónoma de México, Apartado Postal 877, CP 22800 Ensenada, B.C., \\ Mexico
}

Received 12 May 2014 / Accepted 17 June 2014

\section{ABSTRACT}

\begin{abstract}
Context. Carbon and oxygen abundances in stars are important in many fields of astrophysics including nucleosynthesis, stellar structure, evolution of galaxies, and formation of planetary systems. Still, our knowledge of the abundances of these elements in different stellar populations is uncertain because of difficulties in observing and analyzing atomic and molecular lines of $\mathrm{C}$ and $\mathrm{O}$. Aims. Abundances of $\mathrm{C}, \mathrm{O}$, and $\mathrm{Fe}$ are determined for $\mathrm{F}$ and $\mathrm{G}$ main-sequence stars in the solar neighborhood with metallicities in the range $-1.6<[\mathrm{Fe} / \mathrm{H}]<+0.4$ in order to study trends and possible systematic differences in the $\mathrm{C} / \mathrm{Fe}, \mathrm{O} / \mathrm{Fe}$, and $\mathrm{C} / \mathrm{O}$ ratios for thin- and thick-disk stars as well as high- and low-alpha halo stars. In addition, we investigate if there is any connection between $\mathrm{C}$ and $\mathrm{O}$ abundances in stellar atmospheres and the occurrence of planets.

Methods. Carbon abundances are determined from the $\lambda \lambda 5052,5380 \mathrm{CI}$ lines and oxygen abundances from the $\lambda 7774 \mathrm{O}$ I triplet and the forbidden [OI] line at $6300 \AA$. MARCS model atmospheres are applied and non-LTE corrections for the O I triplet are included. Results. Systematic differences between high- and low-alpha halo stars and between thin- and thick-disk stars are seen in the trends of $[\mathrm{C} / \mathrm{Fe}]$ and $[\mathrm{O} / \mathrm{Fe}]$. The two halo populations and thick-disk stars show the same trend of $[\mathrm{C} / \mathrm{O}]$ versus $[\mathrm{O} / \mathrm{H}]$, whereas the thin-disk stars are shifted to higher $[\mathrm{C} / \mathrm{O}]$ values. Furthermore, we find some evidence of higher $\mathrm{C} / \mathrm{O}$ and $\mathrm{C} / \mathrm{Fe}$ ratios in stars hosting planets than in stars for which no planets have been detected.

Conclusions. The results suggest that $\mathrm{C}$ and $\mathrm{O}$ in both high- and low-alpha halo stars and in thick-disk stars are made mainly in massive $\left(M>8 M_{\odot}\right)$ stars, whereas thin-disk stars have an additional carbon contribution from low-mass AGB and massive stars of high metallicity causing a rising trend of the $\mathrm{C} / \mathrm{O}$ ratio with increasing metallicity. However, at the highest metallicities investigated $([\mathrm{Fe} / \mathrm{H}] \simeq+0.4), \mathrm{C} / \mathrm{O}$ does not exceed 0.8 , which seems to exclude formation of carbon planets if proto-planetary disks have the same composition as their parent stars.
\end{abstract}

Key words. stars: abundances - stars: atmospheres - planetary systems - Galaxy: disk - Galaxy: halo

\section{Introduction}

Next to hydrogen and helium, carbon and oxygen are the most abundant elements in the Universe, and their abundances are of high importance in many fields of astrophysics, for example stellar age determinations (Bond et al. 2013), chemical evolution of galaxies (Chiappini et al. 2003; Carigi et al. 2005; Cescutti et al. 2009), and structure of exoplanets (Bond et al. 2010; Madhusudhan 2012).

While it is generally accepted that oxygen is produced by hydrostatic burning in massive stars and then dispersed to the interstellar medium in SNeII explosions (e.g., Kobayashi et al. 2006), the origin of carbon is more uncertain. Both massive $\left(M>8 M_{\odot}\right)$ and low- to intermediate-mass stars probably contribute, but their relative importance and yields are not well known due to

* Based on observations made with the Nordic Optical Telescope and on data products from observations made with ESO Telescopes at the La Silla Paranal Observatory under programs given in Table 2 and in Tables 1 and 2 of Nissen \& Schuster (2010).

$\star \star$ Tables 2-5 are available in electronic form at http://www . aanda. org, and also available at the CDS via anonymous ftp to cdsarc.u-strasbg. fr (130.79.128.5) or via http://cdsarc.u-strasbg.fr/viz-bin/qcat?]/A+A/568/A25 uncertainties about metallicity-dependent mass loss (Meynet \& Maeder 2002; van den Hoek \& Groenewegen 1997).

The nucleosynthesis and Galactic evolution of $\mathrm{C}$ and $\mathrm{O}$ may be studied by determining abundances in F, G, and $\mathrm{K}$ stars with different ages and metallicities. There is, however, still considerable uncertainty about the abundances of $\mathrm{C}$ and $\mathrm{O}$, because the available atomic and molecular lines provide diverging results depending on non-LTE corrections and atmospheric models applied (Asplund 2005). In the case of oxygen, all studies show a rising trend of the oxygen-to-iron ratio as a function of decreasing iron abundance, but the derived level of $[\mathrm{O} / \mathrm{Fe}]^{1}$ among metal-poor halo star ranges from 0.4 dex to $0.8 \mathrm{dex}$, corresponding to more than a factor of two in the $\mathrm{O} / \mathrm{Fe}$ ratio (Nissen et al. 2002; Fulbright \& Johnson 2003; Cayrel et al. 2004; García Pérez 2006; Ramírez et al. 2013). Carbon, on the other hand, follows iron more closely than oxygen, but some studies suggest small deviations from the solar C/Fe ratio in low-metallicity disk and halo stars (Gustafsson et al. 1999; Shi et al. 2002; Reddy et al. 2006; Fabbian et al. 2009; Takeda \& Takada-Hidai 2013).

Additional information about the origin and Galactic evolution of carbon and oxygen may be obtained from differences in

1 For two elements, $X$ and $Y$, with number densities $N_{X}$ and $N_{Y}$, $[X / Y] \equiv \log \left(N_{X} / N_{Y}\right)_{\text {star }}-\log \left(N_{X} / N_{Y}\right)_{\text {Sun }}$. 
$[\mathrm{C} / \mathrm{Fe}],[\mathrm{O} / \mathrm{Fe}]$, and $[\mathrm{C} / \mathrm{O}]$ between stellar populations. Precise abundance studies of $\mathrm{F}$ and $\mathrm{G}$ main-sequence stars in the solar neighborhood have revealed a clear difference in $[\mathrm{O} / \mathrm{Fe}]$ between thin- and thick-disk stars in the metallicity range $-0.7<$ $[\mathrm{Fe} / \mathrm{H}]<-0.2$ (Bensby et al. 2004; Ramírez et al. 2013). A similar difference in $[\mathrm{O} / \mathrm{Fe}]$ between the two populations of highand low-alpha ${ }^{2}$ halo stars identified by Nissen \& Schuster (2010) has been found by Ramírez et al. (2012). In the case of carbon, Reddy et al. (2006) have found evidence of a systematic difference in $[\mathrm{C} / \mathrm{Fe}]$ between thin- and thick-disk stars, but this is not confirmed by Bensby \& Feltzing (2006). For the high- and lowalpha halo stars, Nissen \& Schuster (2014) found indications of a systematic difference in $[\mathrm{C} / \mathrm{Fe}]$, but this should be studied further.

More metal-rich stars, i.e., those having $[\mathrm{Fe} / \mathrm{H}]>-0.2$, also seem to have a bimodal distribution of various abundance ratios. The precise abundances of $1111 \mathrm{~F}$ and $\mathrm{G}$ stars in the solar neighborhood based on HARPS spectra (Adibekyan et al. 2011, 2012) have revealed the existence of a population of metal-rich, alphaelement-enhanced stars having $[\alpha / \mathrm{Fe}] \simeq+0.1$ in contrast to normal thin-disk stars with $[\alpha / \mathrm{Fe}] \simeq+0.0$. Bensby et al. (2014) confirm the existence of these alpha-enhanced stars, but consider them to belong to the thick-disk population. Haywood et al. (2013) also consider the metal-rich, alpha-enhanced stars to belong to the thick-disk sequence in the $[\alpha / \mathrm{Fe}]-[\mathrm{Fe} / \mathrm{H}]$ diagram. They have determined precise ages for the upper main-sequence HARPS stars and find the thick-disk stars to have a well-defined age-metallicity relation ranging from $13 \mathrm{Gyr}$ at $[\mathrm{Fe} / \mathrm{H}] \simeq-1$ to $8 \mathrm{Gyr}$ at $[\mathrm{Fe} / \mathrm{H}] \simeq+0.2$. In contrast, thin-disk stars with $[\mathrm{Fe} / \mathrm{H}]>-0.2$ have ages less than $8 \mathrm{Gyr}$ and a poorly defined age-metallicity relation.

The analysis of HARPS stars by Adibekyan et al. (2012) does not include $\mathrm{C}$ and $\mathrm{O}$ abundances. Hence, it remains to be seen if the two disk populations can be traced in $[\mathrm{C} / \mathrm{Fe}]$ and $[\mathrm{O} / \mathrm{Fe}]$ at metallicities up to $[\mathrm{Fe} / \mathrm{H}] \simeq+0.2$. The abundances of $\mathrm{C}$ and $\mathrm{O}$ are furthermore of great interest in connection with exoplanets. In some recent studies with carbon abundances derived from high-excitation $\mathrm{C} I$ lines and oxygen abundances from the $\lambda 6300$ [O I] line (Delgado Mena et al. 2010; Petigura \& Marcy 2011) the carbon-to-oxygen ratio $^{3}, \mathrm{C} / \mathrm{O}$, in metal-rich $\mathrm{F}$ and $\mathrm{G}$ stars has been found to range from $\sim 0.4$ to $\gtrsim 1.0$, i.e., up to a factor of two higher than the solar ratio, $\mathrm{C} / \mathrm{O}_{\odot} \simeq 0.55$ (Asplund et al. 2009; Caffau et al. 2008, 2010). This has led to suggestions about the existence of exo-planets consisting of carbides and graphite instead of Earth-like silicates (Kuchner \& Seager 2005; Bond et al. 2010). However, an alternative study by Nissen (2013) with oxygen abundances determined from the $\lambda 7774 \mathrm{O}$ I triplet results in a tight, slightly increasing relation between $\mathrm{C} / \mathrm{O}$ and $[\mathrm{Fe} / \mathrm{H}]$ corresponding to $\mathrm{C} / \mathrm{O} \simeq 0.8$ at the highest metallicities $([\mathrm{Fe} / \mathrm{H}]=+0.4)$. This result is supported by a recent study of Teske et al. (2014) of $\mathrm{C} / \mathrm{O}$ ratios in 16 stars with transiting planets. Furthermore, the very low frequency $\left(<10^{-3}\right)$ of carbon stars among $\mathrm{K}$ and $\mathrm{M}$ dwarf suggests that $\mathrm{C} / \mathrm{O}>1$ is also very rare among solar-type stars (Fortney 2012). Still, there is need for further studies of $\mathrm{C} / \mathrm{O}$ ratios in stars hosting planets.

There may be other effects in addition to chemical evolution and population differences, which cause carbon and oxygen abundances to vary among $F$ and $G$ stars. In a very precise

\footnotetext{
2 Alpha refers to the average abundance of the $\alpha$-capture elements, $\mathrm{Mg}, \mathrm{Si}, \mathrm{Ca}$, and $\mathrm{Ti}$.

3 C/O is defined as $N_{\mathrm{C}} / N_{\mathrm{O}}$, where $N_{\mathrm{C}}$ and $N_{\mathrm{O}}$ are the number densities of carbon and oxygen nuclei, respectively. It should not be confused with the solar-normalized logarithmic ratio, $[\mathrm{C} / \mathrm{O}]$.
}

comparison of abundances in the Sun and 11 solar twin stars, Meléndez et al. (2009) found the Sun to have a 20\% depletion of refractory elements like Fe relative to volatile elements like $\mathrm{C}$ and $\mathrm{O}$. They suggest that this may be related to depletion of refractory elements when terrestrial planets formed. Their results are supported by Ramírez et al. (2014), who in a strictly differential abundance analysis for groups of stars with similar $T_{\text {eff }}, \log g$, and $[\mathrm{Fe} / \mathrm{H}]$ values find the slope of $[X / \mathrm{Fe}]$ versus condensation temperature $T_{\mathrm{C}}$ of element $X$ to vary with an amplitude of $\sim 10^{-4}$ dex $\mathrm{K}^{-1}$ corresponding to variations of $\sim 0.1 \mathrm{dex}$ in $[\mathrm{C} / \mathrm{Fe}]$ and $[\mathrm{O} / \mathrm{Fe}]$. Based on HARPS spectra, González Hernández et al. (2010, 2013) find, however, smaller variations of the slope of $[\mathrm{X} / \mathrm{Fe}]$ versus $T_{\mathrm{C}}$ and both positive and negative slopes for ten stars with detected super-Earth planets, which casts doubts about the suggestion that a high volatile-torefractory element ratio can be used as a signature of terrestrial planets. Clearly, more work is needed to explain the small variations in the volatile-to-refractory element ratio and to confirm that such variations really occur.

In this paper we address some of the problems mentioned above by determining precise $\mathrm{C}$ and $\mathrm{O}$ abundances for two samples of $\mathrm{F}$ and $\mathrm{G}$ stars, i.e., 66 disk stars with HARPS and FEROS spectra, and 85 halo and thick-disk stars from Nissen \& Schuster (2010). We aim at getting new information on the evolution of $[\mathrm{C} / \mathrm{Fe}],[\mathrm{O} / \mathrm{Fe}]$, and $[\mathrm{C} / \mathrm{O}]$ in stellar populations and to determine $\mathrm{C} / \mathrm{O}$ ratios for stars with and without detected planets.

\section{Stellar spectra and equivalent widths}

\subsection{The HARPS-FEROS sample of disk stars}

Based on Adibekyan et al. (2012) we have selected 66 mainsequence stars with $5400 \mathrm{~K}<T_{\text {eff }}<6400 \mathrm{~K}$, which have high signal-to-noise (S/N) HARPS and FEROS spectra available in the ESO Science Archive. This sample includes 32 stars with detected planets from Nissen (2013), and 34 new stars many of which have no detected planets. Except for HD 203608 with $[\mathrm{Fe} / \mathrm{H}]=-0.66$, the stars have metallicities in the range $-0.5<$ $[\mathrm{Fe} / \mathrm{H}]<+0.5$, and most of them are thin-disk stars, but a few have thick-disk kinematics.

The HARPS spectra cover a wavelength range from 3800 to $6900 \AA$ with a resolution of $R \simeq 115000$ (Mayor et al. 2003). After combination of many individual spectra of a given star, the S/N exceeds 300 for most of the stars. These spectra were used to measure the equivalent widths (EWs) of the $\lambda \lambda 5052,5380 \mathrm{C}$ I lines and the forbidden [OI] line at $6300 \AA$, if not disturbed by telluric $\mathrm{O}_{2}$ lines. In addition, the EWs of 12 Fe II lines listed in Table 1 were measured from the HARPS spectra.

The FEROS (Kaufer et al. 1999) spectra, which have a resolution of $R \simeq 48000$ and a typical $\mathrm{S} / \mathrm{N}$ of 200 , were used to measure the EWs of the O I triplet lines at $7774 \AA$.

As described in Nissen (2013), the spectra were first normalized with the IRAF continuum task using a low order cubic spline fitting function. Then the IRAF splot task was used to measure equivalent widths by Gaussian fitting relative to local continuum regions selected to be free of lines in the solar spectrum. Care was taken to use the same continuum windows in all stars.

References for the stellar spectra, $\mathrm{S} / \mathrm{N}$, and measured EW values are given in Table 2. In addition, we have used HARPS and FEROS spectra of reflected sunlight from Ceres and Ganymede to represent the solar-flux spectrum. The combined HARPS spectrum has $S / N \simeq 600$ and the FEROS solar-flux 
Table 1. Line data and derived solar abundances.

\begin{tabular}{|c|c|c|c|c|c|c|}
\hline ID & $\begin{array}{c}\text { Wavelength } \\
(\AA)\end{array}$ & $\begin{array}{l}\chi_{\mathrm{exc}} \\
(\mathrm{eV})\end{array}$ & $\log (g f)$ & $\begin{array}{l}E W_{\odot} \\
(\mathrm{m} \AA)\end{array}$ & $\begin{array}{c}A(X)_{\odot}{ }^{a} \\
\text { LTE }^{-1}\end{array}$ & $\begin{array}{c}A(X)_{\odot} \\
\text { non-LTE }\end{array}$ \\
\hline $\mathrm{CI}_{\mathrm{I}}$ & 5052.17 & 7.685 & -1.301 & 35.9 & 8.44 & 8.43 \\
\hline $\mathrm{C}_{\mathrm{I}}$ & 5380.34 & 7.685 & -1.616 & 21.5 & 8.44 & 8.43 \\
\hline [O I] & 6300.31 & 0.000 & -9.720 & $3.7^{b}$ & 8.68 & \\
\hline $\mathrm{OI}_{\mathrm{I}}$ & 7771.94 & 9.146 & 0.369 & 71.8 & 8.88 & 8.66 \\
\hline O I & 7774.17 & 9.146 & 0.223 & 61.8 & 8.86 & 8.66 \\
\hline O I & 7775.39 & 9.146 & 0.002 & 48.6 & 8.84 & 8.67 \\
\hline Fe II & 5414.08 & 3.22 & -3.580 & 28.1 & 7.47 & \\
\hline Fe II & 5425.26 & 3.20 & -3.220 & 42.0 & 7.43 & \\
\hline Fe II & 5991.38 & 3.15 & -3.540 & 31.2 & 7.43 & \\
\hline $\mathrm{Fe}$ II & 6084.11 & 3.20 & -3.790 & 21.0 & 7.45 & \\
\hline $\mathrm{Fe}$ II & 6113.33 & 3.22 & -4.140 & 11.6 & 7.47 & \\
\hline $\mathrm{Fe}$ II & 6149.25 & 3.89 & -2.690 & 36.3 & 7.40 & \\
\hline $\mathrm{Fe}$ II & 6238.39 & 3.89 & -2.600 & 44.3 & 7.51 & \\
\hline $\mathrm{Fe}$ II & 6239.95 & 3.89 & -3.410 & 12.7 & 7.41 & \\
\hline Fe II & 6247.56 & 3.89 & -2.300 & 52.7 & 7.40 & \\
\hline Fe II & 6369.46 & 2.89 & -4.110 & 19.8 & 7.43 & \\
\hline Fe II & 6432.68 & 2.89 & -3.570 & 41.3 & 7.45 & \\
\hline $\mathrm{Fe}$ II & 6456.39 & 3.90 & -2.050 & 63.2 & 7.39 & \\
\hline
\end{tabular}

Notes. ${ }^{(a)}$ For an element $X, A(X) \equiv \log \left(N_{X} / N_{\mathrm{H}}\right)+12.0 .^{\left({ }^{b}\right)}$ This value refers to the $\mathrm{EW}$ of the $[\mathrm{O} \mathrm{I}]$ line after correction for the Ni I contribution to the $[\mathrm{O} \mathrm{I}]-\mathrm{Ni}$ I $\lambda 6300$ blend. The total EW of the blend is $5.4 \mathrm{~m} \AA$.

spectrum has $S / N \simeq 400$ in the $\mathrm{O}$ I-triplet region. The measured EWs are given in Table 1 and are used in a differential modelatmosphere analysis of the stars with respect to the Sun.

\subsection{The UVES-FIES sample of halo and thick-disk stars}

The second sample, for which we have determined $\mathrm{C}$ and $\mathrm{O}$ abundances, consists of high-velocity, $\mathrm{F}$ and $\mathrm{G}$ main-sequence stars in the solar neighborhood from Nissen \& Schuster (2010). These stars have metallicities in the range $-1.6<[\mathrm{Fe} / \mathrm{H}]<-0.4$ and most of them belong to the halo population, i.e. they have space velocities with respect to the local standard of rest (LSR) larger than $180 \mathrm{~km} \mathrm{~s}^{-1}$, but 16 stars with thick-disk kinematics are included. Spectra of stars on the southern sky were acquired from the VLT/UVES archive. They have resolutions $R \simeq 55000$ and $\mathrm{S} / \mathrm{N}$ from 250 to 500 . Northern stars were observed with the FIES spectrograph at the Nordic Optical Telescope at a resolution of $R \simeq 40000$ and with $S / N \simeq 140-200$. Further details are given in Nissen \& Schuster (2010, Tables 1 and 2).

The UVES and FIES spectra include the C I lines at 5052 and $5380 \AA$, but the O I triplet at $7774 \AA$ is not covered. Instead, we have obtained equivalent widths for the O I lines from other sources, first of all Ramírez et al. (2012), who measured EWs for the majority of the Nissen-Schuster stars based on high resolution spectra observed with the HET/HRS, Keck/HIRES, and Magellan/MIKE spectrographs. In addition, we have included EW-values from Nissen \& Schuster (1997) for 15 stars with ESO NTT/EMMI spectra, and from Nissen et al. (2002) for six stars with UVES image slicer spectra. Finally, new EW measurements were carried out for two stars that have FEROS spectra available. Table 3 lists the results with mean values given, if there is more than one source for a star.

As a check of the accuracy of the equivalent width measurements for the O I triplet, Fig. 1 shows a comparison of values from Ramírez et al. (2012) and the other sources mentioned (EMMI, UVES, and FEROS). As seen the agreement is quite

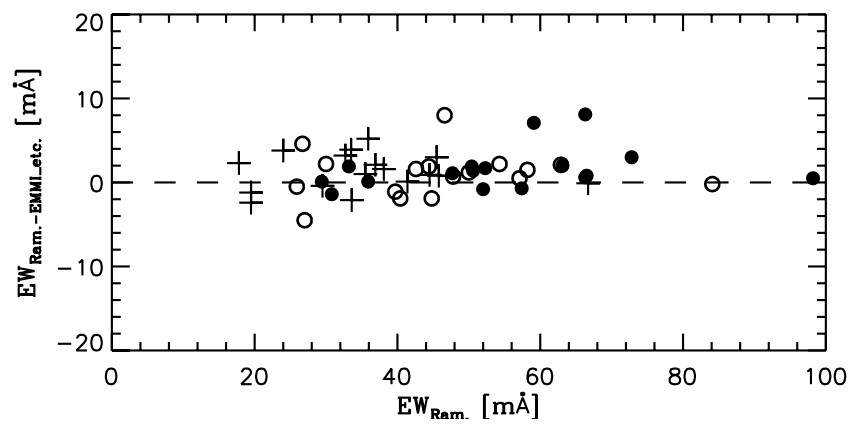

Fig. 1. Comparison of O I equivalent widths from Ramírez et al. (2012) and values based on EMMI, UVES and FEROS spectra. Filled circles refer to O I $\lambda 7771.9$, open circles to O I $\lambda 7774.2$, and crosses to O I $\lambda 7775.4$.

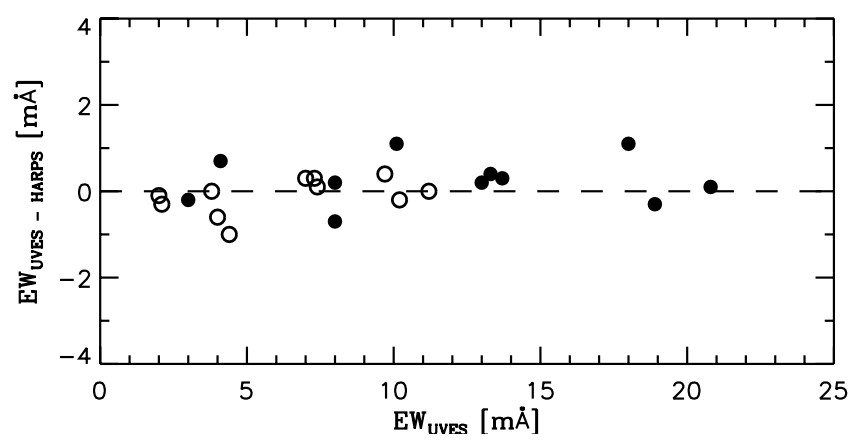

Fig. 2. Comparison of C I equivalent widths measured from UVES and HARPS spectra, respectively. Filled circles refer to C I $\lambda 5052.2$ and open circles to C I $\lambda 5380.3$.

satisfactory. There is a mean difference of $1.3 \mathrm{~m} \AA$ (Ramírez other) with an rms scatter of $2.4 \mathrm{~m} \AA$.

For 11 stars with UVES spectra, HARPS spectra are also available. Figure 2 shows a comparison of the EWs of the C I $\lambda \lambda 5052,5380$ lines measured with the two instruments. As seen, there in an excellent agreement with a mean difference (UVES-HARPS) of $0.1 \mathrm{~m} \AA$ and a rms deviation of only $0.5 \mathrm{~m} \AA$. This good agreement can be ascribed to the high resolution and $\mathrm{S} / \mathrm{N}$ of the two sets of spectra. The FIES spectra have lower resolution and $\mathrm{S} / \mathrm{N}$ resulting in more uncertain $\mathrm{EW}$ measurements for the $\mathrm{C}$ I lines with errors on the order of $2 \mathrm{~m} \AA$.

The weak [O I]-Ni I blend could be measured in UVES spectra of 13 of the most metal-rich halo and thick-disk stars. Five of these stars also have HARPS spectra. The mean difference of the two sets of EWs is $0.0 \mathrm{~m} \AA$ and the rms deviation is $0.4 \mathrm{~m} \AA$.

To illustrate the high quality of the spectra applied, we show in Fig. 3 a region around the $\lambda 5052$ C I line for a low-alpha star, HD 105004, and a high-alpha star, G 05-40, with similar atmospheric parameters and metallicities. As seen, the Fe I lines have the same strengths in the two stars, but the C I line (and a Ti I line) is weaker in the low-alpha star.

\section{Model-atmosphere analysis and non-LTE corrections}

Plane parallel (1D) model atmospheres interpolated to the $T_{\text {eff }}$, $\log g,[\mathrm{Fe} / \mathrm{H}]$, and $[\alpha / \mathrm{Fe}]$ values of the stars were obtained from the MARCS grid (Gustafsson et al. 2008) and the Uppsala program EQWIDTH was used to calculate equivalent widths as a 


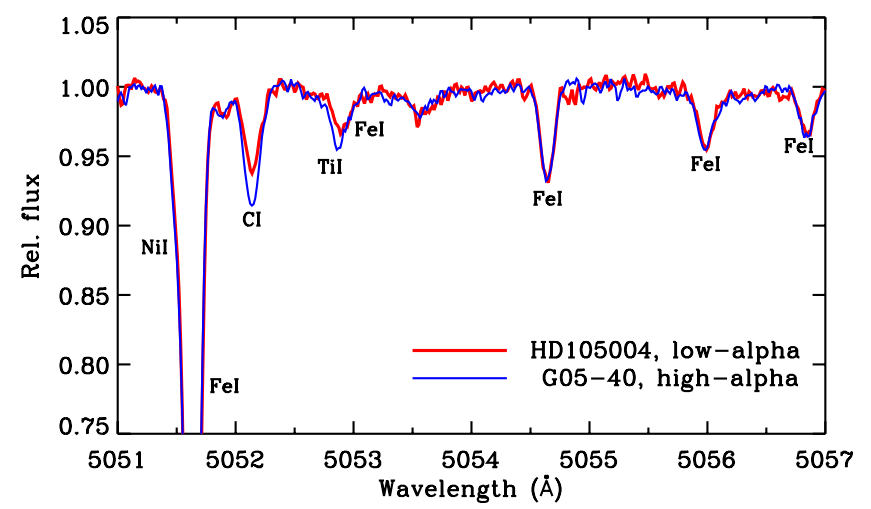

Fig. 3. UVES spectra around the $\lambda 5052 \mathrm{C}$ I line for the low-alpha star, HD 105004 with parameters $\left(T_{\text {eff }}, \log g,[\mathrm{Fe} / \mathrm{H}],[\alpha / \mathrm{Fe}]\right)=(5852 \mathrm{~K}$, $4.35,-0.83,0.14)$, and the high-alpha star, G05-40 with $\left(T_{\text {eff }}, \log g\right.$, $[\mathrm{Fe} / \mathrm{H}],[\alpha / \mathrm{Fe}])=(5892 \mathrm{~K}, 4.20,-0.83,0.31)$.

function of element abundance assuming LTE. By interpolating to the observed EW, we then obtain the LTE abundance corresponding to a given line.

Line data used in the analysis are given in Table 1 . The sources of $g f$-values are Hibbert et al. (1993) for C I lines, Hibbert et al. (1991) for O I lines, and Meléndez \& Barbuy (2009) for Fe II lines. As the analysis is made differentially to the Sun line by line, possible errors in these $g f$-values cancel out. Doppler broadening due to microturbulence is specified by a depth-independent parameter, $\xi_{\text {turb }}$. Collisional broadening caused by neutral hydrogen and helium atoms is based on the Unsöld (1955) approximation with an enhancement factor of two for the C I lines, whereas quantum mechanical calculations of Barklem et al. (2000) and Barklem \& Aspelund-Johansson (2005) is used for the O I and Fe II lines.

Non-LTE corrections calculated by Fabbian et al. (2009) were applied to the oxygen abundances derived from the O I triplet. Fabbian et al. use a model atom with 54 energy levels and adopt electron collision cross sections from Barklem (2007). Inelastic collisions with hydrogen atoms were described by the classical Drawin formula (Drawin 1968) scaled by an empirical factor $S_{\mathrm{H}}$. Calculations were performed for both $S_{\mathrm{H}}=0$ and 1 , which allow us to interpolate the non-LTE corrections to $S_{\mathrm{H}}=$ 0.85 , i.e., the value preferred by Pereira et al. (2009) based on a study of the solar center-to-limb variation of the O I triplet lines.

As seen from Table 1, the solar non-LTE corrections range from -0.22 to -0.17 dex for the three O I lines. According to Fabbian et al. (2009), the corrections depend strongly on $T_{\text {eff }}$; stars cooler than the Sun have less negative non-LTE corrections, and warmer stars have more negative corrections. This leads to very significant differential non-LTE corrections of $[\mathrm{O} / \mathrm{H}]$ for our sample of stars ranging from about $-0.2 \mathrm{dex}$ at $T_{\mathrm{eff}} \simeq 6300 \mathrm{~K}$ to +0.1 dex at $T_{\text {eff }} \simeq 5400 \mathrm{~K}$.

The non-LTE corrections for carbon abundances derived from the C I $\lambda \lambda 5052,5380$ lines are much smaller than those for the OI triplet. In this paper we adopt the calculations of Takeda \& Honda (2005), who assume $S_{\mathrm{H}}=1$. The corrections are -0.01 dex for the Sun and changes only slightly with $T_{\text {eff }}$ and $\log g$.

Furthermore, we note that the $\mathrm{Fe}$ abundance determined from Fe II lines is unaffected by departures from LTE (e.g., Mashonkina et al. 2011; Lind et al. 2012). This is also the case for oxygen abundances derived from the forbidden [O I] line at $6300 \AA$ (e.g. Kiselman 1993). This line is, however, affected by a Ni I blend as further discussed in Sect. 5 .

\section{Stellar parameters}

\subsection{The HARPS-FEROS sample}

For these stars, Adibekyan et al. (2012) have given effective temperatures, $T_{\text {eff }}$, and surface gravities, $\log g$. The values were originally derived from the HARPS spectra by Sousa et al. (2008, $2011 \mathrm{a}, \mathrm{b})$ by requesting that $[\mathrm{Fe} / \mathrm{H}]$ should not have any systematic dependence on excitation potential of the lines and that the same iron abundance is obtained from Fe I and Fe II lines.

As an alternative, we have determined photometric parameters for the HARPS-FEROS sample of disk stars. $T_{\text {eff }}$ is derived from the $(b-y)$ and $(V-K)$ color indices using the calibrations of Casagrande et al. (2010), which are based on $T_{\text {eff }}$ values determined with the infrared flux method (IRFM). $V$ magnitudes and $(b-y)$ were taken from Olsen (1983) and $K$ magnitudes from the 2MASS catalogue (Skrutskie et al. 2006). For nine stars, the 2MASS $K$ value is uncertain due to saturation. In these cases, $T_{\text {eff }}$ has been determined from $(b-y)$ alone. Otherwise we have adopted the mean $T_{\text {eff }}$ derived from $(b-y)$ and $(V-K)$.

The surface gravity was determined from the relation

$\log \frac{g}{g_{\odot}}=\log \frac{M}{M_{\odot}}+4 \log \frac{T_{\mathrm{eff}}}{T_{\mathrm{eff}, \odot}}+0.4\left(M_{\mathrm{bol}}-M_{\mathrm{bol}, \odot}\right)$

where $M$ is the mass of the star and $M_{\text {bol }}$ the absolute bolometric magnitude. HIPPARCos parallaxes (van Leeuwen 2007) are used to derive $M_{V}$. Bolometric corrections were adopted from Casagrande et al. (2010), and stellar masses were obtained by interpolating in the luminosity $-\log T_{\text {eff }}$ diagram between the Yonsei-Yale evolutionary tracks of Yi et al. (2003); see Nissen $\&$ Schuster (2012) for details.

Metallicities were determined from the twelve Fe II lines listed in Table 1. These lines have equivalent widths spanning a range that allows us to determine the microturbulence parameter, $\xi_{\text {turb }}$, from the requirement that the derived $[\mathrm{Fe} / \mathrm{H}]$ should not depend on EW. The analysis is made differentially with respect to the Sun adopting a solar microturbulence of $1.0 \mathrm{~km} \mathrm{~s}^{-1}$.

This procedure of determining stellar parameters has to be iterated until consistency, because the $T_{\text {eff }}$ calibrations, bolometric corrections and mass determinations depend on $[\mathrm{Fe} / \mathrm{H}]$.

All stars have distances less than $60 \mathrm{pc}$ according to their HIPPARCos parallaxes. We may therefore assume that the observed $(b-y)$ and $(V-K)$ indices and hence $T_{\text {eff }}$ are not affected by interstellar reddening.

The rms dispersion of the difference of $T_{\text {eff }}$ determined from $(b-y)$ and $(V-K)$, respectively, is $62 \mathrm{~K}$. From this we estimate that the mean photometric temperature, $T_{\text {eff }}($ phot $)=1 / 2 \times$ $\left(T_{\mathrm{eff}}(b-y)+T_{\mathrm{eff}}(V-K)\right)$ is determined with an internal onesigma error of $\sim 30 \mathrm{~K}$. At a given metallicity, this high precision is confirmed by comparing the photometric $T_{\text {eff }}$ values with the spectroscopic temperatures of Adibekyan et al. (2012), who also quote an error of $30 \mathrm{~K}$; the rms scatter of $\Delta T_{\text {eff }}=T_{\text {eff }}$ (phot) $T_{\text {eff }}$ (spec) in metallicity intervals of $0.2 \mathrm{dex}$ is about $40 \mathrm{~K}$. As seen from Fig. 4, there is, however, a systematic trend of $\Delta T_{\text {eff }}$ as a function of metallicity. The difference between the two sets of temperatures decreases from about $+50 \mathrm{~K}$ at $[\mathrm{Fe} / \mathrm{H}]=-0.4$ to about $-60 \mathrm{~K}$ at $[\mathrm{Fe} / \mathrm{H}]=+0.4$.

Because of the small error in the HIPPARCos parallaxes, the estimated error in $\log g$ (HIP) is about 0.05 dex. This small statistical error is supported by the comparison of photometric and spectroscopic gravities shown in Fig. 5; the rms scatter of $\Delta \log g=\log g(\mathrm{HIP})-\log g$ (spec) in $T_{\text {eff }}$ intervals of $200 \mathrm{~K}$ is only $0.06 \mathrm{dex}$, but there is a trend of $\Delta \log g$ as a function of $T_{\text {eff }}$ ranging from about $+0.1 \mathrm{dex}$ at $T_{\mathrm{eff}}=5400 \mathrm{~K}$ to about $-0.15 \mathrm{dex}$ 
P. E. Nissen et al.: Carbon and oxygen abundances in stellar populations

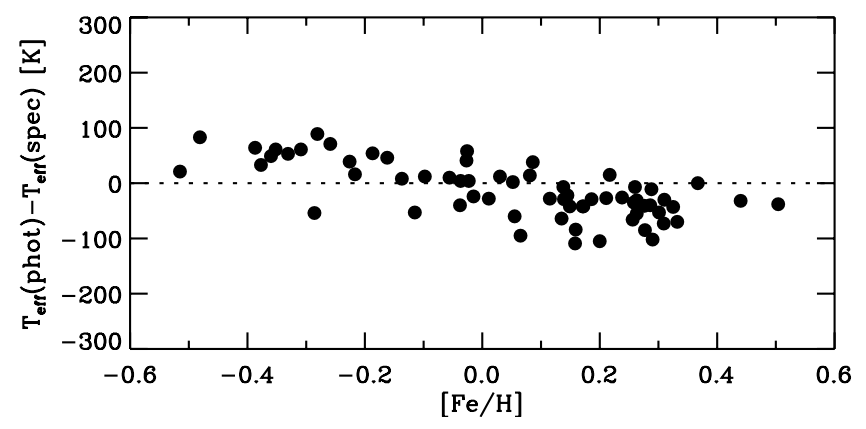

Fig. 4. Comparison of photometric temperatures for the HARPSFEROS stars derived in this paper and the spectroscopic temperatures derived by Sousa et al. (2008, 2011a,b)

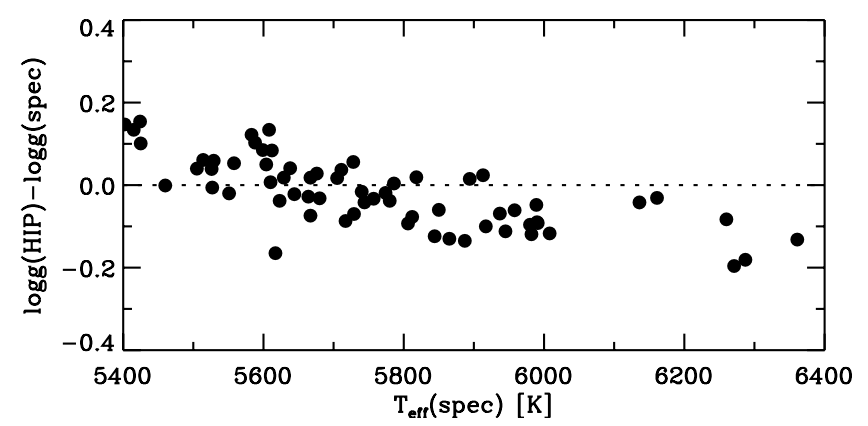

Fig. 5. Comparison of surface gravities for the HARPS-FEROS stars derived via HIPPARCos parallaxes in this paper and the spectroscopic gravities derived by Sousa et al. $(2008,2011 \mathrm{a}, \mathrm{b})$.

at the highest temperatures. This trend is not easily explained; according to Lind et al. (2012) non-LTE effects on the ionization balance of $\mathrm{Fe}$ (used to determine the spectroscopic gravities) are almost negligible for the effective temperatures, gravities and metallicities of the present HARPS-FEROS sample of stars. The same problem is discussed by Bensby et al. (2014), who note that dwarf stars with spectroscopic gravities have a flat distribution of $\log g$ as a function of $T_{\text {eff }}$. In contrast, gravities derived from HIPPARCos parallaxes show an increasing trend of $\log g$ when $T_{\text {eff }}$ decreases from $6500 \mathrm{~K}$ to $5000 \mathrm{~K}$ in agreement with predictions from isochrones. Hence, it looks like the HIPPARCos gravities are more reliable, but it is puzzling why the spectroscopic gravities have systematic errors. As discussed by Bensby et al. (2014), the small departures from LTE for the Fe I lines predicted with 1D models (Lind et al. 2012) cannot explain the problem. Perhaps one may be able to solve the problem when full 3D, non-LTE calculations of the ionization balance of Fe becomes available.

In view of the problems with the spectroscopic gravities, we prefer to apply the photometric values based on HIPPARcos parallaxes. For consistency we then also adopt the photometric $T_{\text {eff }}$ values based on the $(b-y)$ and $(V-K)$ colors and the Casagrande et al. (2010) calibration. These parameters are given in Table 4 together with the derived values of $[\mathrm{Fe} / \mathrm{H}]$ and $\xi_{\text {turb }}$, which agree well with those of Adibekyan et al. (2012) despite of the differences in $T_{\text {eff }}$ and $\log g$. Mean differences (this paper - Adibekyan) and rms scatters are: $\Delta[\mathrm{Fe} / \mathrm{H}]=0.015 \pm 0.04$ and $\Delta \xi_{\text {turb }}=0.03 \pm 0.09 \mathrm{~km} \mathrm{~s}^{-1}$.

\subsection{The UVES-FIES sample}

This sample consists of halo and thick-disk stars with distances up to $350 \mathrm{pc}$, which means that interstellar reddening may affect

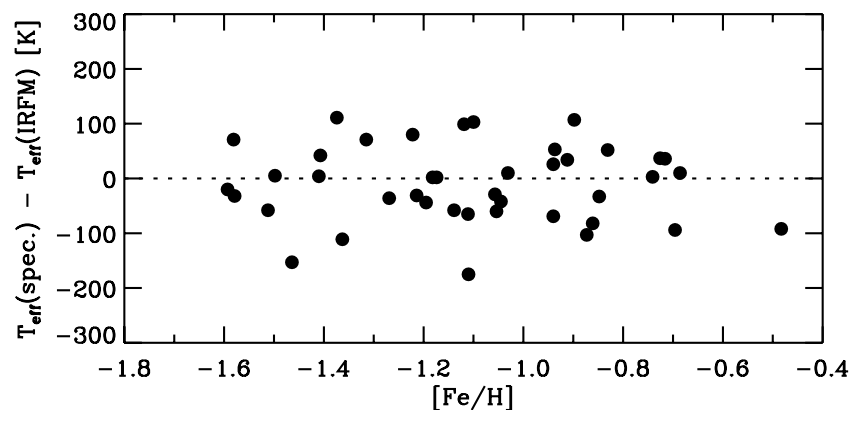

Fig. 6. Comparison of spectroscopic temperatures derived in this paper for the UVES-FIES sample and temperatures determined by Casagrande et al. (2010) with the IRFM method.

the effective temperature determined from color indices and that in most cases the HIPPARCos parallax has a too large error to be used to determine the surface gravity. Hence, it is not possible to determine precise photometric values of $T_{\text {eff }}$ and $\log g$. Instead, we have followed the method applied by Nissen \& Schuster (2010, 2011), who first determined $T_{\text {eff }}$ and $\log g$ for two nearby, unreddened thick-disk stars, HD 22879 and HD 76932, with the photometric method described in the previous section. These standard stars are analyzed relative to the Sun using lines having EWs less than about $100 \mathrm{~m} \AA$ in the solar spectrum. An inverted abundance analysis then yields $g f$-values for the full set of lines given in Nissen \& Schuster (2011, Table 3). Using these $g f$-values, a model atmosphere analysis makes it possible to determine spectroscopic values of $T_{\text {eff }}$ and $\log g$ as well as highprecision abundances relative to the standard stars.

In Nissen \& Schuster (2010, 2011), the calibrations of Ramírez \& Meléndez (2005) were used to determine $T_{\text {eff }}$ of the standard stars. The more accurate calibrations by Casagrande et al. (2010) show, however, a systematic offset of about $+100 \mathrm{~K}$ in $T_{\text {eff }}$ relative to the Ramírez \& Meléndez values. We have, therefore, increased the $T_{\text {eff }}$ values of the standard stars by $100 \mathrm{~K}$, and repeated the spectroscopic analysis of the other stars, i.e., determined $T_{\text {eff }}$ from the excitation balance of weak $(E W<50 \mathrm{~m} \AA)$ Fe I lines, $\log g$ from the $\mathrm{Fe} \mathrm{I} / \mathrm{Fe}$ II ionization balance, and $\xi_{\text {turb }}$ by requesting that the derived $[\mathrm{Fe} / \mathrm{H}]$ has no systematic dependence on EW for Fe I lines. The updated parameters are given in Table 5 for 85 stars for which $\mathrm{C}$ and/or $\mathrm{O}$ abundances have been determined ${ }^{4}$. Relative to the parameter values given in Nissen \& Schuster $(2010,2011)$, there are small changes in $\log g$ ranging from +0.03 dex for the standard stars to about +0.08 dex for the coolest stars. $[\mathrm{Fe} / \mathrm{H}]$ is decreased by approximately 0.02 dex for all stars, whereas $[\alpha / \mathrm{Fe}]$ is practically the same, i.e., changes are within \pm 0.005 dex.

As a check of the spectroscopic values of $T_{\text {eff }}$, we have compared with IRFM values from Casagrande et al. (2010) for a subsample of 41 stars in common. As seen from Fig. 6, there is no significant trend of $\Delta T_{\text {eff }}=T_{\text {eff }}$ (spec) $-T_{\text {eff }}$ (IRFM) as a function of $[\mathrm{Fe} / \mathrm{H}]$, nor is there any trend of $\Delta T_{\text {eff }}$ with $T_{\text {eff }}$. The mean value of $\Delta T_{\text {eff }}$ is $-10 \mathrm{~K}$ with an rms scatter of $\pm 70 \mathrm{~K}$. The largest contribution to this scatter arises from the error in $T_{\text {eff }}$ (IRFM), which is on the order of $\pm 60 \mathrm{~K}$. These numbers suggest that the error in $T_{\text {eff }}(\mathrm{spec})$ is on the order of $\pm 35 \mathrm{~K}$.

The spectroscopic gravities have been tested by comparing with gravities based on HIPPARCos parallaxes for 24 stars $^{5}$,

\footnotetext{
4 The C and s-process rich CH-subgiant, G24-25 (Liu et al. 2012) is not included.

5 The two standard stars are not included in this comparison, because their spectroscopic and photometric gravities agree by definition.
} 
Table 6. Errors in abundance ratios of the metal-rich star HD $196050^{a}$ caused by errors in model atmosphere parameters and equivalent widths.

\begin{tabular}{lcccccccc}
\hline \hline & $\sigma[\mathrm{Fe} / \mathrm{H}]$ & $\sigma[\mathrm{C} / \mathrm{H}]$ & $\sigma[\mathrm{O} / \mathrm{H}]_{6300}{ }^{b}$ & $\sigma[\mathrm{O} / \mathrm{H}]_{7774}{ }^{c}$ & $\sigma[\mathrm{C} / \mathrm{Fe}]$ & $\sigma[\mathrm{O} / \mathrm{Fe}]_{6300}{ }^{b}$ & $\sigma[\mathrm{O} / \mathrm{Fe}]_{7774}{ }^{c} \sigma[\mathrm{C} / \mathrm{O}]_{7774}{ }^{c}$ \\
\hline$\sigma\left(T_{\text {eff }}\right)= \pm 30 \mathrm{~K}$ & $\mp 0.011$ & $\mp 0.020$ & \pm 0.002 & $\mp 0.035$ & $\mp 0.009$ & \pm 0.013 & $\mp 0.024$ & \pm 0.015 \\
$\sigma(\log g)= \pm 0.05 \mathrm{dex}$ & \pm 0.020 & \pm 0.017 & \pm 0.023 & \pm 0.021 & $\mp 0.003$ & \pm 0.003 & \pm 0.001 & $\mp 0.004$ \\
$\sigma[\mathrm{Fe} / \mathrm{H}]= \pm 0.03 \mathrm{dex}$ & \pm 0.008 & \pm 0.000 & \pm 0.010 & \pm 0.001 & $\mp 0.008$ & \pm 0.002 & $\mp 0.007$ & $\mp 0.001$ \\
$\sigma\left(\xi_{\text {turb }}\right)= \pm 0.06 \mathrm{~km} \mathrm{~s}^{-1}$ & $\mp 0.014$ & $\mp 0.003$ & \pm 0.000 & $\mp 0.005$ & \pm 0.011 & \pm 0.014 & \pm 0.009 & \pm 0.002 \\
$\sigma(E W)$ & \pm 0.010 & \pm 0.020 & \pm 0.040 & \pm 0.025 & \pm 0.022 & \pm 0.041 & \pm 0.027 & \pm 0.032 \\
\hline$\sigma$ (total) & \pm 0.030 & \pm 0.033 & \pm 0.047 & \pm 0.048 & \pm 0.028 & \pm 0.045 & \pm 0.038 & \pm 0.036 \\
\hline
\end{tabular}

Notes. ${ }^{(a)}\left(T_{\text {eff }}, \log g,[\mathrm{Fe} / \mathrm{H}]\right)=(5862 \mathrm{~K}, 4.22,+0.26)^{(b)}$ The oxygen abundance is based on the [O I $] \lambda 6300$ line. ${ }^{(c)}$ The oxygen abundance is based on the O I $\lambda 7774$ triplet.

Table 7. Errors in abundance ratios of the metal-poor star HD $199289^{a}$ caused by errors in model atmosphere parameters and equivalent widths.

\begin{tabular}{lcccccccc}
\hline \hline & $\sigma[\mathrm{Fe} / \mathrm{H}]$ & $\sigma[\mathrm{C} / \mathrm{H}]$ & $\sigma[\mathrm{O} / \mathrm{H}]_{6300}{ }^{b}$ & $\sigma[\mathrm{O} / \mathrm{H}]_{7774}{ }^{c}$ & $\sigma[\mathrm{C} / \mathrm{Fe}]$ & $\sigma[\mathrm{O} / \mathrm{Fe}]_{6300}{ }^{b}$ & $\sigma[\mathrm{O} / \mathrm{Fe}]_{7774}{ }^{c} \sigma[\mathrm{C} / \mathrm{O}]_{7774}{ }^{c}$ \\
\hline$\sigma\left(T_{\text {eff }}\right)= \pm 35 \mathrm{~K}$ & $\mp 0.002$ & $\mp 0.018$ & \pm 0.012 & $\mp 0.034$ & $\mp 0.016$ & \pm 0.014 & $\mp 0.032$ & \pm 0.016 \\
$\sigma(\log g)= \pm 0.06 \mathrm{dex}$ & \pm 0.022 & \pm 0.024 & \pm 0.024 & \pm 0.023 & \pm 0.002 & \pm 0.002 & \pm 0.001 & \pm 0.001 \\
$\sigma[\mathrm{Fe} / \mathrm{H}]= \pm 0.03 \mathrm{dex}$ & \pm 0.002 & $\mp 0.002$ & \pm 0.004 & $\mp 0.001$ & $\mp 0.004$ & \pm 0.002 & $\mp 0.003$ & $\mp 0.001$ \\
$\sigma\left(\xi_{\text {turb }}\right)= \pm 0.06 \mathrm{~km} \mathrm{~s}^{-1}$ & $\mp 0.008$ & \pm 0.000 & \pm 0.000 & $\mp 0.003$ & \pm 0.008 & \pm 0.008 & \pm 0.005 & \pm 0.003 \\
$\sigma(E W)$ & \pm 0.015 & \pm 0.045 & \pm 0.050 & \pm 0.025 & \pm 0.047 & \pm 0.052 & \pm 0.029 & \pm 0.051 \\
\hline$\sigma$ (total $)$ & \pm 0.028 & \pm 0.054 & \pm 0.057 & \pm 0.048 & \pm 0.050 & \pm 0.055 & \pm 0.044 & \pm 0.054 \\
\hline
\end{tabular}

Notes. ${ }^{(a)}\left(T_{\text {eff }}, \log g,[\mathrm{Fe} / \mathrm{H}]\right)=(5915 \mathrm{~K}, 4.30,-1.05) .{ }^{(b)}$ The oxygen abundance is based on the [O I $] \lambda 6300$ line. ${ }^{(c)}$ The oxygen abundance is based on the O I $\lambda 7774$ triplet.

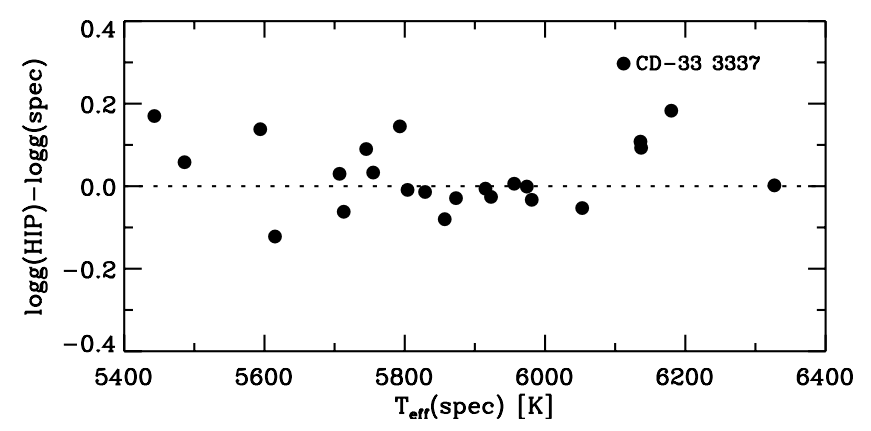

Fig. 7. Comparison of photometric gravities derived via HIPPARcos parallaxes and spectroscopic gravities derived from Fe I and Fe II lines for a subsample of the UVES-FIES stars having parallax errors less than $10 \%$.

which have no detectable interstellar $\mathrm{NaD}$ lines (indicating that interstellar reddening is negligible) and parallaxes with an error of less than $10 \%$. As seen from Fig. 7, there is a satisfactory agreement between the two sets of gravities although one star, $\mathrm{CD}-333337$, shows a large deviation. If this outlier is excluded, the mean of $\Delta \log g=\log g$ (HIP) $-\log g$ (spec) is 0.03 dex with an rms scatter of \pm 0.085 dex. With an estimated average error of $\pm 0.06 \mathrm{dex}$ for $\log g$ (HIP), the error of $\log g$ (spec) becomes $\pm 0.06 \mathrm{dex}$. If CD -333337 is included, the scatter of $\Delta \log g$ raises to \pm 0.10 dex and the error of $\log g$ (spec) to \pm 0.08 dex.

Interestingly, there is no indication of a trend of $\Delta \log g$ as a function of $T_{\text {eff }}$ like the trend seen in Fig. 4 for the more metalrich disk stars. Thus, it seems that we can use spectroscopic values of $\log g$ for the UVES-FIES sample of halo and thickdisk stars without worrying about systematic errors as a function of $T_{\text {eff }}$.

\section{Carbon and oxygen abundances}

The derived LTE and non-LTE values of $[\mathrm{C} / \mathrm{H}]$ and $[\mathrm{O} / \mathrm{H}]$ are given in Tables 4 and 5 . In the case of $[\mathrm{O} / \mathrm{H}]$ we list both the value derived from the $\mathrm{O}$ I triplet and the value based on the [OI] $\lambda 6300$ line, if measured. Most of the disk stars have $[\mathrm{O} / \mathrm{H}]_{6300}$ available, whereas the $[\mathrm{OI}]$ line is too weak for the majority of halo stars to be detectable.

\subsection{Statistical errors}

Statistical errors of the various abundance ratios have been determined for two representative stars: a disk star, HD 196050 , with $[\mathrm{Fe} / \mathrm{H}]=+0.26$ and a metal-poor star from the UVES-FIES sample, HD 199289, with $[\mathrm{Fe} / \mathrm{H}]=-1.05$. As seen from Tables 6 and 7, $[\mathrm{O} / \mathrm{H}]_{6300}$ derived from the $[\mathrm{OI}] \lambda 6300$ line is only slightly affected by the uncertainty in $T_{\text {eff }}$, whereas $[\mathrm{O} / \mathrm{H}]_{7774}$ derived from the $\mathrm{O}$ I triplet lines is sensitive to the error in $T_{\text {eff }} .[\mathrm{Fe} / \mathrm{H}],[\mathrm{C} / \mathrm{H}]$, and $[\mathrm{O} / \mathrm{H}]$ are all significantly affected by the error in $\log g$, but this dependence cancels out for $[\mathrm{C} / \mathrm{Fe}],[\mathrm{O} / \mathrm{Fe}]$, and $[\mathrm{C} / \mathrm{O}]$. Furthermore, we note that the errors in $[\mathrm{Fe} / \mathrm{H}]$ and $\xi_{\text {turb }}$ are of minor importance for the derived abundance ratios.

The errors of $[\mathrm{Fe} / \mathrm{H}],[\mathrm{C} / \mathrm{H}]$, and $[\mathrm{O} / \mathrm{H}]_{7774}$ arising from the uncertainty of the measured equivalent widths have been estimated from the line-to-line scatter of the derived abundances. In the case of $[\mathrm{O} / \mathrm{H}]_{6300}$, the EW-error corresponds to an uncertainty of the equivalent width of the $\lambda 6300$ [O I] line of $\pm 0.4 \mathrm{~m} \AA$ as estimated in Sect. 2.1.

The total errors listed in the last rows of Tables 6 and 7 are calculated by adding the individual errors in quadrature. The small estimated error of $[\mathrm{C} / \mathrm{Fe}]( \pm 0.028 \mathrm{dex})$ in Table 6 is due 
to the extremely high $\mathrm{S} / \mathrm{N}$ of the HARPS spectra, and the small sensitivity of $[\mathrm{C} / \mathrm{Fe}]$ to uncertainties in the atmospheric parameters. For the UVES-FIES sample the error of $[\mathrm{C} / \mathrm{Fe}]$ is larger, because of the weakness of the $\mathrm{C}$ I lines, especially in spectra of metal-poor halo stars belonging to the low-alpha population. In some of the figures in Sect. 6, data will be shown with individual error bars.

\subsection{Systematic errors; the $[\mathrm{O} / \mathrm{H}]_{7774}-[\mathrm{O} / \mathrm{H}]_{6300}$ discrepancy}

In addition to statistical errors, we have to consider possible systematic errors of the abundances, especially in the case of oxygen abundances derived from the O I $\lambda 7774$ lines, because of the large non-LTE corrections and high sensitivity to $T_{\text {eff }}$. In order to reveal any problems, we have therefore compared oxygen abundances derived from the $\mathrm{O}$ I triplet with $\mathrm{O}$ abundances resulting from the forbidden oxygen line at $6300 \AA$.

As discussed in detail by Allende-Prieto et al. (2001), the absorption feature at $6300.3 \AA$ is a blend of the [O I] line and a Ni I line, which makes it slightly asymmetric. From the best fit of the blend with profiles calculated from a 3D model atmosphere of the Sun, they derived a solar oxygen abundance of $A(\mathrm{O})_{\odot}=8.69$. Later, Johansson et al. (2003) measured an experimental oscillator strength of the Ni I line, $\log g f=-2.11 \pm 0.05$. When using this value and a solar nickel abundance of $A(\mathrm{Ni})_{\odot}=$ 6.17 (determined from 17 weak Ni I lines), Scott et al. (2009) obtained $A(\mathrm{O})_{\odot}=8.69$ from a 3D analysis. Repeating the analysis of the Ni I lines with a 1D MARCS model of the Sun, we obtain a $\mathrm{Ni}$ abundance of 6.15 and predict an equivalent width of $1.7 \mathrm{~m} \AA$ for the Ni I line in the $\lambda 6300$ blend. After subtracting this value from the measured $\mathrm{EW}$ of the [OI] - Ni I blend

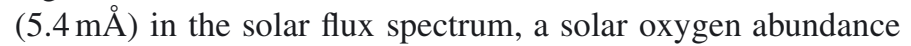
of 8.68 is obtained, which agrees well with the $3 \mathrm{D}$ value.

For the stars we have first calculated the EW of the blending $\mathrm{Ni}$ I line using $\mathrm{Ni}$ abundances corresponding to the $[\mathrm{Ni} / \mathrm{Fe}]$ values of Adibekyan et al. (2012) for the HARPS-FEROS sample and those of Nissen \& Schuster (2010) for the UVES-FIES sample. The measured EW of the [O I] - Ni I blend is then corrected for the contribution from the $\mathrm{Ni}$ I line before the oxygen abundance is determined.

A comparison of non-LTE oxygen abundances derived from the $\mathrm{O} I-$ triplet and $\mathrm{O}$ abundances from the $[\mathrm{OI}]$ line is shown in Fig. 8. The average of $\Delta[\mathrm{O} / \mathrm{H}]=[\mathrm{O} / \mathrm{H}]_{7774}-[\mathrm{O} / \mathrm{H}]_{6300}$ is 0.010 dex with an rms scatter of $\pm 0.07 \mathrm{dex}$, which corresponds well to the errors estimated in Tables 6 and 7. As seen, there is, however, a systematic deviation for the coolest and most metal-rich stars on the order of $\Delta[\mathrm{O} / \mathrm{H}] \sim+0.10$, i.e., the triplet provides higher oxygen abundances than the $[\mathrm{O} \mathrm{I}] \lambda 6300$ line. A similar deviation was found by Teske et al. (2013) for the planet hosting star $55 \mathrm{Cnc}$ having $T_{\mathrm{eff}}=5350 \mathrm{~K}, \log g=4.44$, and $[\mathrm{Fe} / \mathrm{H}]=+0.34$. They obtained $[\mathrm{O} / \mathrm{H}]_{7774} \simeq 0.22$ to 0.27 (depending on which non-LTE corrections were adopted) and $[\mathrm{O} / \mathrm{H}]_{6300} \simeq 0.08$.

There are several possible explanations of the discrepancy between $[\mathrm{O} / \mathrm{H}]$ derived from the $\mathrm{O}$ I triplet and the [O I] $\lambda 6300$ line. Given that $[\mathrm{Ni} / \mathrm{Fe}]$ increases slightly and $[\mathrm{O} / \mathrm{Fe}]$ decreases with increasing $[\mathrm{Fe} / \mathrm{H}]$, the $\mathrm{Ni}$ I line makes up a large fraction (up to $60 \%$ ) of the [O I] - Ni I blend in spectra of metalrich stars, whereas the corresponding fraction in the solar spectrum is $\sim 30 \%$. These estimates are based on $\log g f=-2.11$ for the Ni I line as determined by Johansson et al. (2003). If $\log g f$ is decreased by for example $0.15 \mathrm{dex}$, the importance of the Ni I line decreases, and the solar oxygen abundance derived from
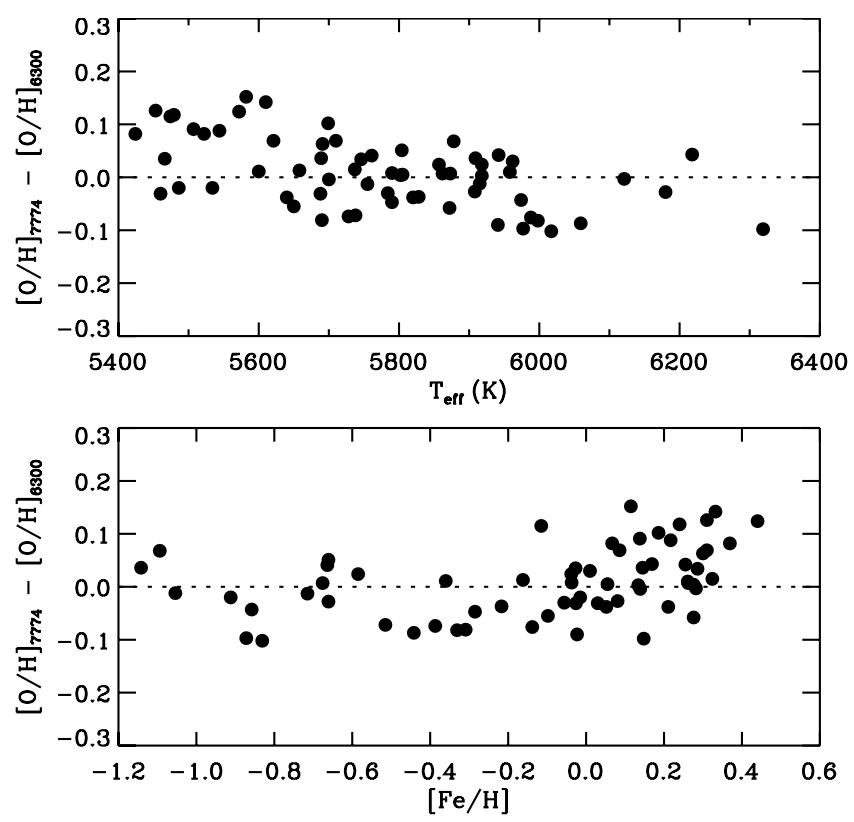

Fig. 8. Differences of non-LTE oxygen abundances derived from the OI $\lambda 7774$ triplet and oxygen abundances obtained from the [O I] $\lambda 6300$ line versus $T_{\text {eff }}$ and $[\mathrm{Fe} / \mathrm{H}]$.

the $[\mathrm{O}$ I] $\lambda 6300$ line increases from $A(\mathrm{O})=8.68$ to 8.74 . For the cool, metal-rich stars in our sample the effect is even larger resulting in an increase in $[\mathrm{O} / \mathrm{H}]_{6300}$ of 0.05 dex to 0.10 dex, which removes most of the discrepancy seen in Fig. 8. A decrease in $\log g f$ of 0.15 dex is, however, three times larger than the error given by Johansson et al. (2003). A lower $g f$-value would, on the other hand, decrease the puzzling difference between oxygen abundances derived from the [O I] lines at 6300 and $6363 \AA$ in spectra of dwarf stars (Caffau et al. 2013). Hence, we think that this possibility should be kept open.

One may ask if problems with the non-LTE corrections for the O I triplet could lead to too high $[\mathrm{O} / \mathrm{H}]$ values compared to those derived from the [O I] $\lambda 6300$ line. The corrections of Fabbian et al. (2009) have a strong dependence of $T_{\text {eff }}$ leading to positive corrections of $[\mathrm{O} / \mathrm{H}]$ for dwarf stars cooler than the Sun. If no corrections are applied, i.e., LTE is assumed, the discrepancy shown in Fig. 8 for the cool, metal-rich stars in fact disappears, but instead there is a clear discrepancy for stars with $T_{\text {eff }}>6000 \mathrm{~K}$, and the scatter of $[\mathrm{O} / \mathrm{H}]_{7774}-[\mathrm{O} / \mathrm{H}]_{6300}$ increases from 0.07 dex to $0.09 \mathrm{dex}$. So, this is not a viable solution. There may, however, be other ways to solve the problem. Schuler et al. (2004, 2006) found that oxygen abundances derived from the $\mathrm{O}$ I triplet show a strong rise with decreasing effective temperature for dwarf stars having $T_{\text {eff }}<5450 \mathrm{~K}$ in the Pleiades and Hyades open clusters. At $T_{\text {eff }}=5000 \mathrm{~K}$ there is an overabundance of $\sim 0.25$ dex relative to the oxygen abundance in the range $5450 \mathrm{~K}<T_{\text {eff }}<6100 \mathrm{~K}$, and at $T_{\text {eff }}=4500 \mathrm{~K}$ the overabundance has increased to $\sim 0.75$ dex. Schuler et al. (2006) suggest that this may be related to the presence of hot and cool spots on the stellar surface with the hot spots making a relative large contribution to the EWs of the O I triplet in the cool Pleiades and Hyades stars. This effect may play a role for the coolest stars in our sample by contributing to the overabundance of $[\mathrm{O} / \mathrm{H}]_{7774}$ relative to $[\mathrm{O} / \mathrm{H}]_{6300}$, although our stars are on average older and probably less active than the Pleiades and Hyades stars.

A difference in oxygen abundances derived from 3D model atmospheres and 1D models is another potential problem. As 
discussed by Nissen et al. (2002), 3D corrections are more important for the [OI] $\lambda 6300$ line than the O I triplet, because the [OI] line is formed in the upper layers of the atmosphere, where $3 \mathrm{D}$-effects have the largest impact on the temperature structure. The dependence of $3 \mathrm{D}$ corrections on $T_{\text {eff }}$ is however small,

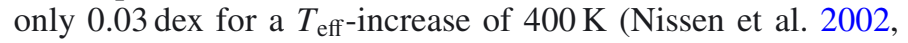
Table 6) and goes in the wrong direction to diminish the discrepancy between $[\mathrm{O} / \mathrm{H}]_{7774}$ and $[\mathrm{O} / \mathrm{H}]_{6300}$ at low $T_{\text {eff }}$.

We conclude from this discussion that there is no obvious explanation of the $[\mathrm{O} / \mathrm{H}]_{7774}-[\mathrm{O} / \mathrm{H}]_{6300}$ discrepancy for the cool, metal-rich stars in our sample, and that the problem could be with both $[\mathrm{O} / \mathrm{H}]_{7774}$ and $[\mathrm{O} / \mathrm{H}]_{6300}$. In the following section we shall, therefore, discuss results based on both sets of oxygen abundances.

\section{Results and discussion}

In this section, we show how $[\mathrm{C} / \mathrm{Fe}],[\mathrm{O} / \mathrm{Fe}]$, and $[\mathrm{C} / \mathrm{O}]$ change as a function of increasing metallicity, $[\mathrm{Fe} / \mathrm{H}]$, for the four populations of stars identified in the solar neighborhood, and discuss how these trends may be explained in terms of Galactic chemical evolution and differences in origin of the various populations. We also investigate, if there are any systematic differences in the abundance ratios between stars hosting planets and stars for which no planets have been detected, and if there is evidence of a cosmic scatter in $[\mathrm{C} / \mathrm{Fe}]$ and $[\mathrm{O} / \mathrm{Fe}]$ for solar analog stars corresponding to the variations in the volatile-to-refractory element ratio claimed in some recent works (e.g. Meléndez et al. 2009; Ramírez et al. 2014).

\subsection{Populations}

As seen from Tables 4 and 5, stars have been classified into four different populations. For the HARPS-FEROS sample, the $[\alpha / \mathrm{Fe}]-[\mathrm{Fe} / \mathrm{H}]$ diagram of Adibekyan et al. (2013) is used to divide stars into thin- and thick-disk populations depending on whether they lie below or above the dividing line shown in their Fig. 1. At $[\mathrm{Fe} / \mathrm{H}]<-0.2$, there is a gab in $[\alpha / \mathrm{Fe}]$ between the two populations, so a chemical separation is more clear than a kinematical separation, because thin- and thick-disk stars have considerable overlap in the Galactic velocity components. For the more metal-rich stars with $[\mathrm{Fe} / \mathrm{H}]>-0.2$, there is no gab between the thin- and thick-disk sequences in the $[\alpha / \mathrm{Fe}]-[\mathrm{Fe} / \mathrm{H}]$ diagram, so the population classification is less clear. Haywood et al. (2013) have, however, shown that the metal-rich, alphaenhanced stars are older than the metal-rich thin-disk stars and that they form a smooth extension of the age-metallicity relation for the metal-poor thick-disk stars (see Fig. 10 in Haywood et al. 2013).

Stars in the UVES-FIES sample were classified by Nissen $\&$ Schuster (2010). If the total space velocity with respect to the LSR is larger than $180 \mathrm{~km} \mathrm{~s}^{-1}$, a star is considered to be a halo star. As shown in Fig. 1 of Nissen \& Schuster (2010), these stars have a bimodal distribution in the $[\alpha / \mathrm{Fe}]-[\mathrm{Fe} / \mathrm{H}]$ diagram allowing a classification into high- and low-alpha halo stars. The two halo populations are also well separated in other abundance ratios such as $\mathrm{Na} / \mathrm{Fe}$ and $\mathrm{Ni} / \mathrm{Fe}$ with the low-alpha stars having abundances ratios similar to those of dwarf galaxies (Tolstoy et al. 2009). Furthermore, the two populations have different kinematical properties and ages, i.e., the low-alpha stars have larger space velocities and more negative (retrograde) $V$ velocity components than the high-alpha stars, and they are on average younger by $2-3$ Gyr (Schuster et al. 2012). Altogether, this

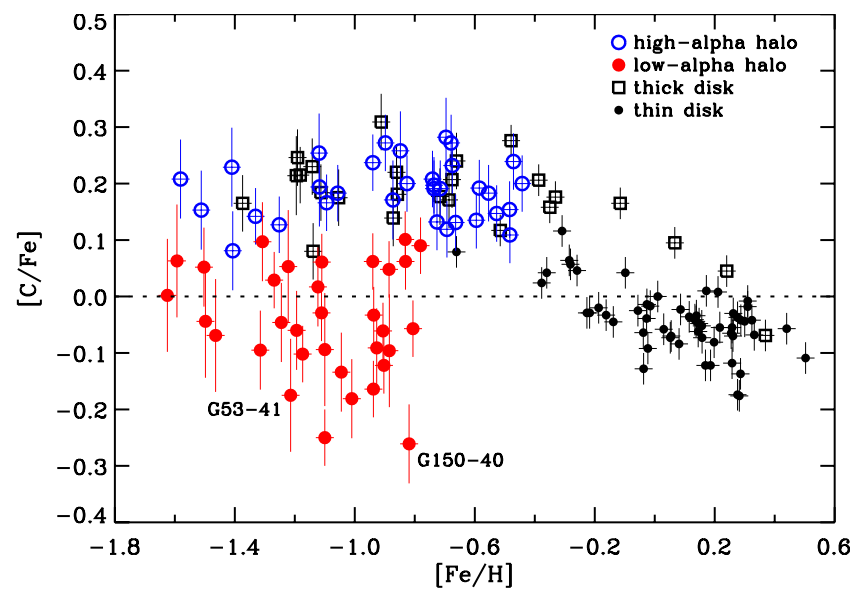

Fig. 9. $[\mathrm{C} / \mathrm{Fe}]$ versus $[\mathrm{Fe} / \mathrm{H}]$ for stars analyzed in this paper. The two Na-rich halo stars are marked.

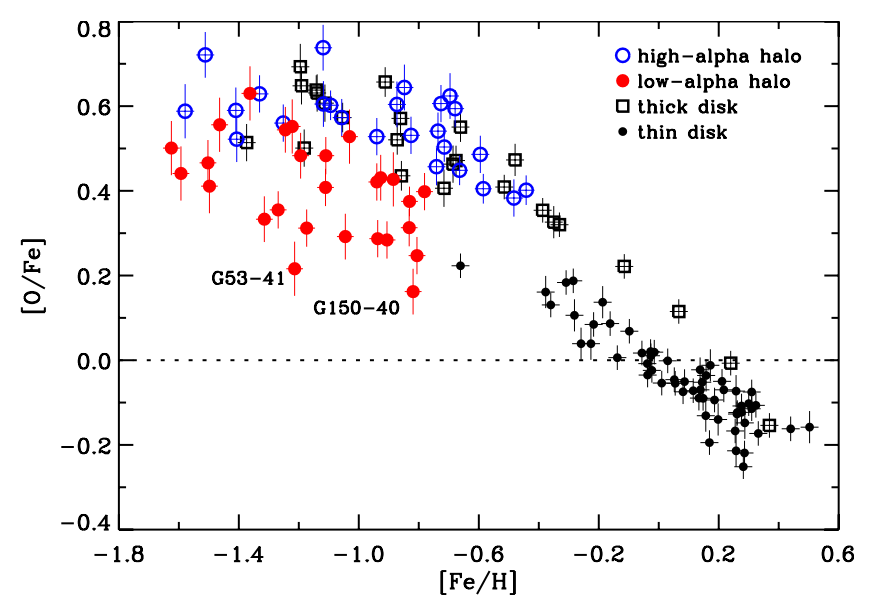

Fig. 10. $[\mathrm{O} / \mathrm{Fe}]$ versus $[\mathrm{Fe} / \mathrm{H}]$. For stars with both $[\mathrm{O} / \mathrm{H}]_{6300}$ and $[\mathrm{O} / \mathrm{H}]_{7774}$ available, the average oxygen abundance has been applied.

suggests that the low-alpha stars have been accreted from dwarf galaxies, whereas the high-alpha stars may have formed in situ during a dissipative collapse of proto-Galactic gas clouds.

The UVES-FIES sample includes 16 stars, which were classified by Nissen \& Schuster (2010) as belonging to the thick disk based on their kinematics. All of these stars fall above the dividing line in the $[\alpha / \mathrm{Fe}]-[\mathrm{Fe} / \mathrm{H}]$ diagram of Adibekyan et al. (2013), so they would also be classified as thick-disk according to their chemical properties.

\subsection{The $[\mathrm{C} / \mathrm{Fe}]$ and $[\mathrm{O} / \mathrm{Fe}]$ trends}

The trends of $[\mathrm{C} / \mathrm{Fe}]$ and $[\mathrm{O} / \mathrm{Fe}]$ as a function of $[\mathrm{Fe} / \mathrm{H}]$ are shown in Figs. 9 and 10, respectively, with different symbols for the four populations discussed. In Fig. 10 we have applied the average oxygen abundance derived from the $\mathrm{O}$ I triplet and the $[\mathrm{OI}] \lambda 6300$ line if both lines were measured. Individual error bars are given; they are relatively large in $[\mathrm{C} / \mathrm{Fe}]$ for the lowalpha halo stars due to the weakness of the $\mathrm{C}$ I lines.

As seen from Figs. 9 and 10, there are systematic differences between thin- and thick-disk stars. The group of thin-disk stars around $[\mathrm{Fe} / \mathrm{H}] \sim-0.3$ falls below the thick-disk sequence in both $[\mathrm{C} / \mathrm{Fe}]$ and $[\mathrm{O} / \mathrm{Fe}]$, and the thin-disk star HD 203608 at $[\mathrm{Fe} / \mathrm{H}]=-0.66$ also falls below. Furthermore, we note that the two metal-rich, alpha-enhanced stars with $[\mathrm{Fe} / \mathrm{H}]$ between -0.15 and +0.15 clearly fall above the thin-disk sequence, and lie on 
P. E. Nissen et al.: Carbon and oxygen abundances in stellar populations

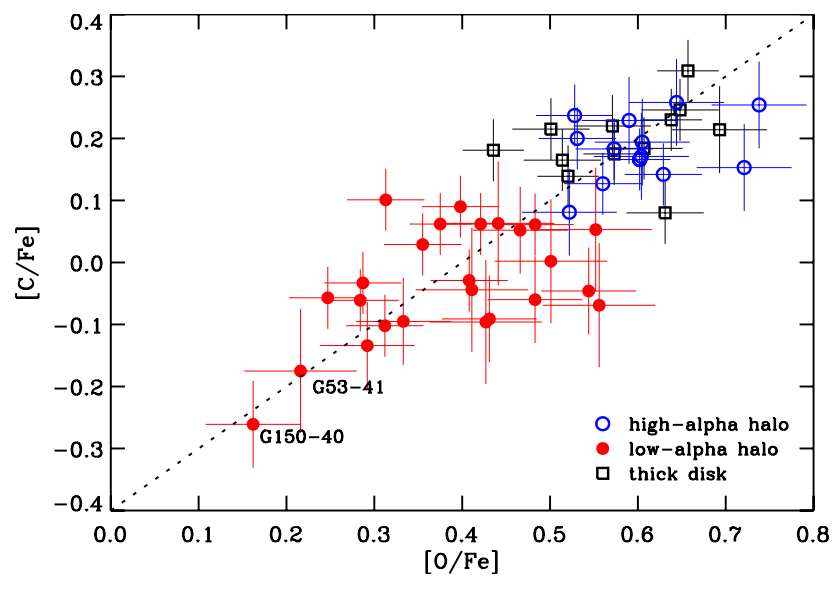

Fig. 11. $[\mathrm{C} / \mathrm{Fe}]$ versus $[\mathrm{O} / \mathrm{Fe}]$ for stars with $[\mathrm{Fe} / \mathrm{H}]<-0.8$. For stars with both $[\mathrm{O} / \mathrm{H}]_{6300}$ and $[\mathrm{O} / \mathrm{H}]_{7774}$ available, the average oxygen abundance has been applied.

an extension of the more metal-poor thick-disk sequence. The third and the fourth metal-rich thick-disk stars with $[\mathrm{Fe} / \mathrm{H}]$ between +0.2 and +0.4 do not deviate significantly from the thindisk stars suggesting that the two populations merge in $[\mathrm{C} / \mathrm{Fe}]$ and $[\mathrm{O} / \mathrm{Fe}]$ at the highest metallicities.

The high-alpha halo stars with $[\mathrm{Fe} / \mathrm{H}]<-0.8$ are distributed around a plateau of $[\mathrm{C} / \mathrm{Fe}] \simeq 0.2$ in Fig. 9 and $[\mathrm{O} / \mathrm{Fe}] \simeq 0.6$ in Fig. 10, whereas the low-alpha stars are shifted downwards with a decreasing trend as a function of increasing metallicity. In the case of $[\mathrm{O} / \mathrm{Fe}]$, this confirms the result of Ramírez et al. (2012), although their $[\mathrm{O} / \mathrm{Fe}]$ values tend to be slightly lower. Furthermore, the trends of $[\mathrm{C} / \mathrm{Fe}]$ and $[\mathrm{O} / \mathrm{Fe}]$ resemble that of $[\mathrm{Mg} / \mathrm{Fe}]$ shown in Nissen \& Schuster (2010) except for a constant offset; the high-alpha halo stars have a plateau at $[\mathrm{Mg} / \mathrm{Fe}] \simeq 0.35$.

Figure 11 shows that $[\mathrm{C} / \mathrm{Fe}]$ and $[\mathrm{O} / \mathrm{Fe}]$ are well correlated and that the amplitude of the variations is about the same for the two abundance ratios, i.e., the data are well fitted with a line having a slope of one. Interestingly, the two stars with the lowest $[\mathrm{C} / \mathrm{Fe}]$ and $[\mathrm{O} / \mathrm{Fe}]$ values, $\mathrm{G} 53-41$ and $\mathrm{G} 150-40$, are Na-rich (Nissen \& Schuster 2010). As discussed by Ramírez et al. (2012), they share the Na-O abundance anomaly of second generation stars born in globular clusters (Carretta et al. 2009).

The different trends of high- and low-alpha stars in Figs. 9 and 10 may be explained if the two populations were formed in systems with different star formation rates. According to this scenario, the high-alpha stars formed in regions with such a high star formation rate that mainly massive stars exploding as Type II SNe contributed to the chemical enrichment up to $[\mathrm{Fe} / \mathrm{H}] \simeq-0.6$ at which metallicity Type Ia SNe started to contribute $\mathrm{Fe}$ causing $[\mathrm{O} / \mathrm{Fe}]$ to decrease. The low-alpha stars, on the other hand, originate in regions with a slow chemical evolution so that Type Ia SNe started to contribute iron at $[\mathrm{Fe} / \mathrm{H}] \simeq-1.6$, or at an even lower metallicity. Bursts of star formation, as those shown in dwarf galaxies, could explain the low $[\mathrm{C} / \mathrm{Fe}]$ and $[\mathrm{O} / \mathrm{Fe}]$ present in low-alpha stars. These values are even lower, if Fe occurs between consecutive bursts (Carigi et al. 2002). The two Na-rich stars, G 53-41 and G 150-40, are exceptions as they have probably been formed in globular clusters as mentioned above.

As an alternative explanation of low-alpha halo stars, Kobayashi et al. (2014) suggest that they were formed in regions where the nucleosynthesis contribution of massive $M>25 M_{\odot}$ core collapse supernovae is missing due to stochastic variations of the IMF. These stochastic effects are more important

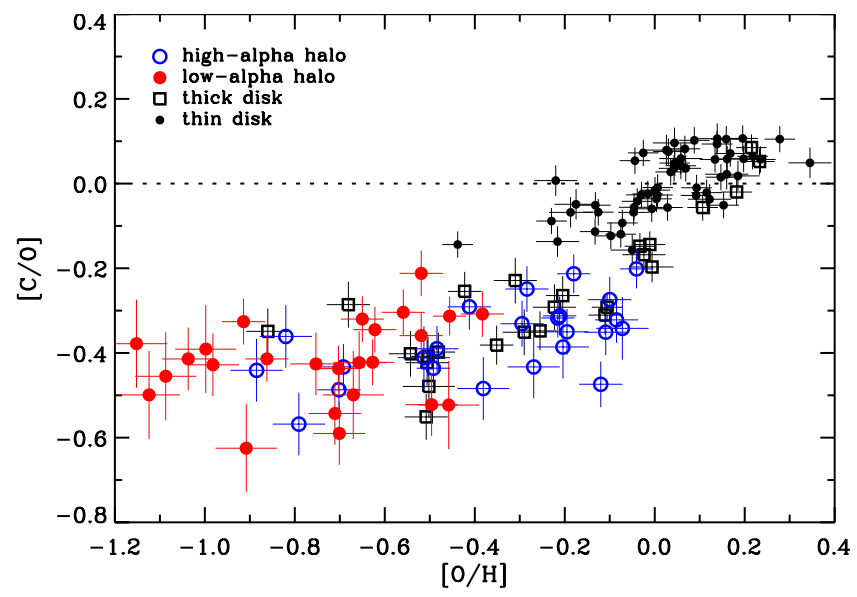

Fig. 12. $[\mathrm{C} / \mathrm{O}]$ versus $[\mathrm{O} / \mathrm{H}]$. For stars with both $[\mathrm{O} / \mathrm{H}]_{6300}$ and $[\mathrm{O} / \mathrm{H}]_{7774}$ available, the average oxygen abundance has been applied.

in dwarf galaxies, due to the low content of available gas to form stars (Carigi \& Hernandez 2008). This explanation is based on the yield calculations of Kobayashi et al. (2006) showing that the $\mathrm{O} / \mathrm{Fe}$ and $\alpha / \mathrm{Fe}$ ratio is significantly lower for $13-25 M_{\odot}$ supernovae than in the case of $M>25 M_{\odot}$ supernovae. The hypothesis also explains why $\mathrm{Mn} / \mathrm{Fe}$ is not enhanced in the low-alpha stars (Nissen \& Schuster 2011), which one might have expected if Type Ia SNe contribution of iron-peak elements is the explanation of the low-alpha stars. It is, however, difficult to see, how this hypothesis can explain why the difference in $[\mathrm{C} / \mathrm{Fe}]$, $[\mathrm{O} / \mathrm{Fe}]$, and $[\alpha / \mathrm{Fe}]$ between high- and low-alpha stars increases as a function of increasing metallicity, which fact has a natural explanation with a delayed contribution of Fe from Type Ia SNe.

As seen from Figs. 9 and 10, thick-disk stars show the same trends of $[\mathrm{C} / \mathrm{Fe}]$ and $[\mathrm{O} / \mathrm{Fe}]$ as high-alpha halo stars. It is not possible to distinguish between these two populations from a chemical point of view. The thick-disk stars differ in kinematics from the high-alpha halo stars by having total space velocities, $V_{\text {total }}<120 \mathrm{~km} \mathrm{~s}^{-1}$ with respect to the LSR, whereas the high-alpha halo stars were selected to have $V_{\text {total }}>180 \mathrm{~km} \mathrm{~s}^{-1}$. It could be that both populations have been formed during a dissipative collaps of proto-Galactic gas clouds with each new generation of stars formed in increasingly flatter and more rotationally supported spheroids.

\subsection{The $[C / O]-[C / H]$ diagram}

The $[\mathrm{C} / \mathrm{O}]-[\mathrm{O} / \mathrm{H}]$ diagram shown in Fig. 12 is particular useful when discussing the origin and Galactic evolution of carbon. Given that oxygen is exclusively produced in massive stars on a relatively short timescale, $\sim 10^{7} \mathrm{yr}$, the change in $[\mathrm{C} / \mathrm{O}]$ as a function of $[\mathrm{O} / \mathrm{H}]$ depends on the yields and timescales of carbon production in various types of stars (Chiappini et al. 2003; Akerman et al. 2004; Carigi et al. 2005; Cescutti et al. 2009; Carigi \& Peimbert 2011).

As seen from Fig. 12, there is no systematic shift in [C/O] between the high- and low-alpha halo populations; the stars distribute around a plateau of $[\mathrm{C} / \mathrm{O}] \simeq-0.45$ when $[\mathrm{O} / \mathrm{H}]<-0.4$. This result came as a surprise. Assuming that the low $[\mathrm{C} / \mathrm{Fe}]$ and $[\mathrm{O} / \mathrm{Fe}]$ in low-alpha stars are due to Fe produced by Type Ia SNe, we had expected that low- and intermediate-mass AGB stars also had enough time to contribute carbon and raise $[\mathrm{C} / \mathrm{O}]$ in the lowalpha stars to higher values than in high-alpha stars. The explanation may be that intermediate-mass (4-8 $M_{\odot}$ ) AGB stars 
contribute very little to carbon (Kobayashi et al. 2011), and that the evolution timescale of low-mass (1-3 $\left.M_{\odot}\right)$ AGB stars, which do have a high carbon yield according to Kobayashi et al., is longer than the timescale for enriching the low-alpha stars with Fe from Type Ia SNe. Hence, it seems that carbon in high- and low-alpha halo stars as well as thick-disk stars with $[\mathrm{O} / \mathrm{H}]<-0.4$ was made mainly in high-mass stars $\left(M>8 M_{\text {Sun }}\right)$ with a $\mathrm{C} / \mathrm{O}$ yield ratio corresponding to $[\mathrm{C} / \mathrm{O}] \simeq-0.45$.

For the high-alpha halo and thick-disk stars, $[\mathrm{C} / \mathrm{O}]$ begins to rise at $[\mathrm{O} / \mathrm{H}] \sim-0.4$ and reaches $[\mathrm{C} / \mathrm{O}] \sim 0.0$ at $[\mathrm{O} / \mathrm{H}] \sim+0.2$. According to Cescutti et al. (2009), this increase can be explained as due to a metallicity dependent carbon yield of massive stars, but Akerman et al. (2004) and Carigi et al. (2005) ascribe the rise of $[\mathrm{C} / \mathrm{O}]$ to low-mass $\left(1-3 M_{\odot}\right)$ AGB stars of low metallicity. The thin-disk stars lie on a different $[\mathrm{C} / \mathrm{O}]$ sequence shifted by $\sim 0.2$ dex to higher $[\mathrm{C} / \mathrm{O}]$ values. To explain this, carbon produced in both low-mass and massive stars has to be included. The model by Carigi et al. (2005) suggests that approximately half of the carbon in the more metal-rich thin-disk stars has come from low-mass stars and half from massive stars. A similar conclusion is reached by Cescutti et al. (2009). Still, it remains to be seen if chemical evolution models can reproduce the observed $[\mathrm{C} / \mathrm{O}]$ trends in detail.

The offset between thin- and thick-disk stars in the $[\mathrm{C} / \mathrm{O}]-[\mathrm{O} / \mathrm{H}]$ diagram has previously been found by Bensby \& Feltzing (2006) in a study where the $\mathrm{C}$ and $\mathrm{O}$ abundances were derived from the forbidden [C I] $\lambda 8727$ and [O I] $\lambda 6300$ lines. Here it is confirmed with $\mathrm{C}$ and $\mathrm{O}$ abundances based on highexcitation permitted lines.

\subsection{The $\mathrm{C} / \mathrm{O}$ ratio in stars with planets}

As mentioned in the introduction, there is much interest in the $\mathrm{C} / \mathrm{O}$ ratio of stars hosting planets, because this ratio may have important effects on the structure and composition of the planets. High ratios $(\mathrm{C} / \mathrm{O}>0.8)$ were found in a significant fraction (10-15\%) of F and G dwarf stars by Delgado Mena et al. (2010) and Petigura \& Marcy (2011) based on oxygen abundances derived from the forbidden [O I] $\lambda 6300$ line, but this was not confirmed by Nissen (2013), who derived oxygen abundances from the O I triplet at $7774 \AA$.

In this paper we have included a number of stars with no detection of planets. $\mathrm{C} / \mathrm{O}$ as a function of $[\mathrm{Fe} / \mathrm{H}]$ for thin-disk stars is shown in Fig. 13 with oxygen abundances derived from either the O I triplet at $7774 \AA$ (upper panel) or the [O I] $\lambda 6300$ line (lower panel). From linear regression, $y=A+B x$, we obtain

$\mathrm{C} / \mathrm{O}_{7774}=0.559( \pm 0.009)+0.287( \pm 0.039)[\mathrm{Fe} / \mathrm{H}]$

with a standard deviation of \pm 0.061 and

$\mathrm{C} / \mathrm{O}_{6300}=0.557( \pm 0.012)+0.529( \pm 0.055)[\mathrm{Fe} / \mathrm{H}]$

with a standard deviation of \pm 0.068 .

In both cases, the zero-point (at $[\mathrm{Fe} / \mathrm{H}]=0.0$ ) is close to the solar value, $(\mathrm{C} / \mathrm{O})_{\odot}=0.58$, determined from HARPS-FEROS spectra of reflected sunlight from Ceres and Ganymede. The slope of $\mathrm{C} / \mathrm{O}_{6300}$ is, however, higher than that of $\mathrm{C} / \mathrm{O}_{7774}$, which is connected to the systematic differences between oxygen abundances derived from the $\mathrm{O}$ I triplet and the forbidden line discussed in Sect. 5. In any case, both fits correspond to $\mathrm{C} / \mathrm{O}$ values below 0.8 up to a metallicity of $[\mathrm{Fe} / \mathrm{H}] \simeq+0.4$, and only two stars have $\mathrm{C} / \mathrm{O}_{6300}$ above 0.8 , which may be statistical deviations. Hence, we confirm the conclusion of Nissen (2013) that

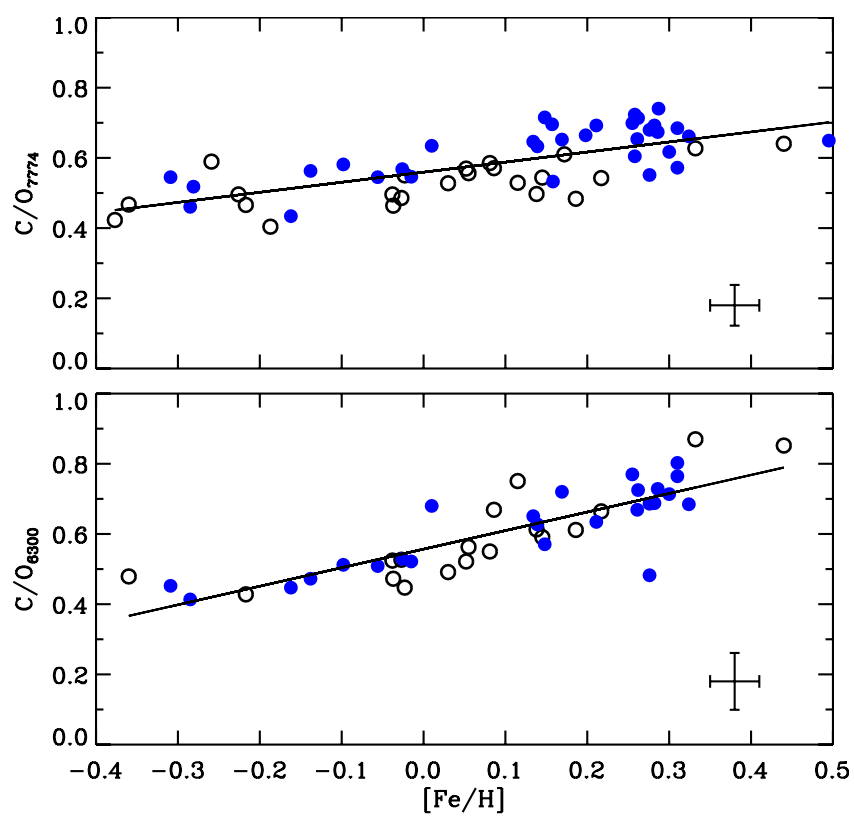

Fig. 13. $\mathrm{C} / \mathrm{O}$ ratio versus $[\mathrm{Fe} / \mathrm{H}]$ for thin-disk stars. Stars proven to have planets (see Table 4) are plotted with filled (blue) circles. In the upper panel, $\mathrm{O}$ abundances have been determined from the $\mathrm{O}$ I triplet and in the lower panel from the [O I] $\lambda 6300$ line. The lines show the relations in Eqs. (2) and (3), respectively

there is no evidence of the existence of stars with $\mathrm{C} / \mathrm{O}$ values above 0.8 , i.e., the critical limit for the formation of carbon planets (Bond et al. 2010).

The dispersions in $\mathrm{C} / \mathrm{O}$ around the fitted lines in Fig. 13 correspond well to the errors of [C/O] estimated in Table 6. Stars with planets detected ${ }^{6}$ tend, however, to lie above the fitted line in $\mathrm{C} / \mathrm{O}_{7774}$; if only stars with planets are included in the regression, the zero-point of the fit becomes $A_{7774}=0.586 \pm 0.013$ compared to $A_{7774}=0.525 \pm 0.013$ for stars without planets. This difference is statistically significant at a 3-sigma level. On the other hand, a smaller difference is found in the case of $\mathrm{C} / \mathrm{O}_{6300}$. Here, the zero-points of the fits are $A_{6300}=0.562 \pm 0.016$ for stars with planets and $A_{6300}=0.552 \pm 0.017$ for stars without planets.

\subsection{Scatter of $[\mathrm{C} / \mathrm{Fe}]$ and $[\mathrm{O} / \mathrm{Fe}]$ among solar analog stars}

In a recent high-precision abundance work, Ramírez et al. (2014) found the slope of abundance ratios $[X / \mathrm{Fe}]$ versus condensation temperature, $T_{\mathrm{C}}$, of element $X$ to vary with an amplitude of $\sim 10^{-4}$ dex $\mathrm{K}^{-1}$. These variations were found for late F-type dwarfs, metal-rich solar analogs, and solar-twin stars. While the $50 \%$ condensation temperatures of $\mathrm{C}$ and $\mathrm{O}$ are 40 and $180 \mathrm{~K}$, respectively, it is $1330 \mathrm{~K}$ for $\mathrm{Fe}$ in a solar-system composition gas (Lodders 2003). Hence, the results of Ramírez et al. imply that $[\mathrm{C} / \mathrm{Fe}]$ and $[\mathrm{O} / \mathrm{Fe}]$ should vary with an amplitude of approximately $0.1 \mathrm{dex}$, which magnitude may be detectable as a cosmic scatter in the $[\mathrm{C} / \mathrm{Fe}]$ and $[\mathrm{O} / \mathrm{Fe}]$ trends for our sample of $\mathrm{F}$ and $\mathrm{G}$ dwarfs stars. Furthermore, the study of Meléndez et al. (2009) showed the Sun to have a high volatile-to-refractory ratio (corresponding to $[\mathrm{C} / \mathrm{Fe}] \sim[\mathrm{O} / \mathrm{Fe}] \sim 0.05$ ) relative to the ratio in 11 solar twins, which they suggest may be due to depletion of refractory elements when terrestrial planets formed.

6 Based on the April 2014 version of the Exoplanet Orbit Database at http://exoplanets . org described by Wright et al. (2011). 
P. E. Nissen et al.: Carbon and oxygen abundances in stellar populations

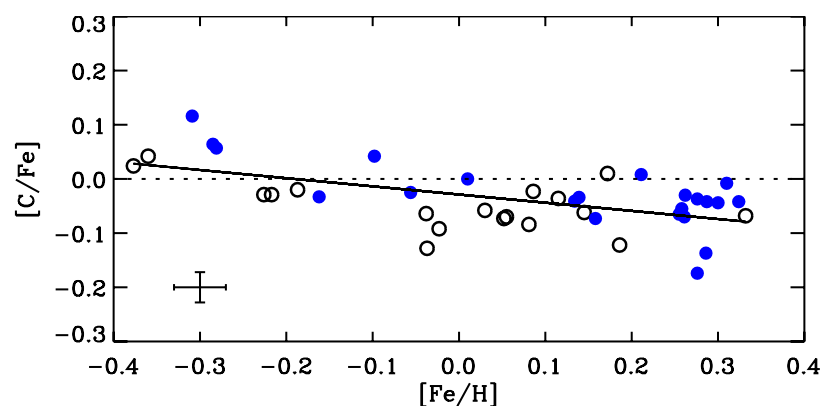

Fig. 14. $[\mathrm{C} / \mathrm{Fe}]$ versus $[\mathrm{Fe} / \mathrm{H}]$ for solar analog stars. Stars proven to have planets are plotted with filled (blue) circles. The line shows the relation in Eq. (4).

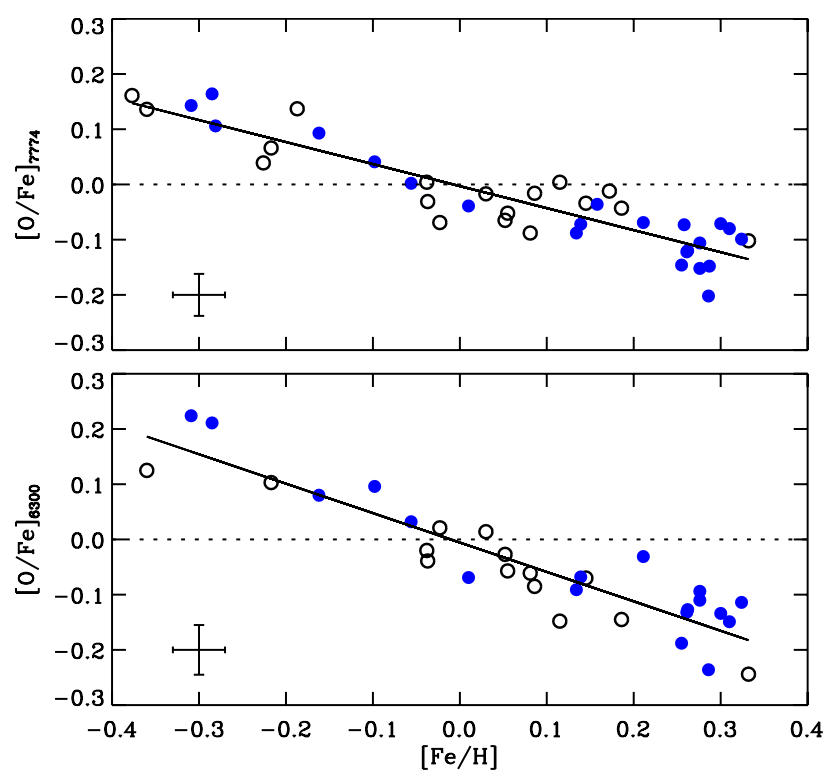

Fig. 15. $[\mathrm{O} / \mathrm{Fe}]$ versus $[\mathrm{Fe} / \mathrm{H}]$ for solar analog stars. Stars proven to have planets are plotted with filled (blue) circles. In the upper panel, O abundances have been determined from the $\mathrm{O}$ I triplet and in the lower panel from the [OI] $\lambda 6300$ line. The lines correspond to Eqs. (5) and (6), respectively

In order to test these intriguing results, we have selected a sample of 40 solar "analog" stars, defined as thin-disk stars with effective temperatures within $\pm 200 \mathrm{~K}$ from the solar $T_{\text {eff }}$ and metallicities in the range $-0.4<[\mathrm{Fe} / \mathrm{H}]<+0.4$. The surface gravity of this sample ranges from $\log g=3.90$ to 4.62 , but the large majority $(N=32)$ of the stars have $\log g>4.20$. The means of the atmospheric parameters are $\left\langle T_{\text {eff }}\right\rangle=5760 \mathrm{~K}$ and $\langle\log g\rangle=4.35$, i.e. close to the solar parameters.

Figure 14 shows a plot of $[\mathrm{C} / \mathrm{Fe}]$ versus $[\mathrm{Fe} / \mathrm{H}]$ for these solar analogs. From linear regression we obtain

$[\mathrm{C} / \mathrm{Fe}]=-0.029( \pm 0.008)-0.151( \pm 0.035)[\mathrm{Fe} / \mathrm{H}]$

with a standard deviation $\sigma[\mathrm{C} / \mathrm{Fe}]=0.047$.

The upper panel of Fig. 15 shows the relation between $[\mathrm{O} / \mathrm{Fe}]_{7774}$ and $[\mathrm{Fe} / \mathrm{H}]$ for the sample of 40 solar analog stars and in the lower panel $[\mathrm{O} / \mathrm{Fe}]_{6300}$ versus $[\mathrm{Fe} / \mathrm{H}]$ is shown for the subsample of 32 solar analogs, which have $\mathrm{O}$ abundances determined from the $[\mathrm{O} \mathrm{I}] \lambda 6300$ line. The linear regressions are

$[\mathrm{O} / \mathrm{Fe}]_{7774}=-0.003( \pm 0.006)-0.399( \pm 0.029)[\mathrm{Fe} / \mathrm{H}]$

with a standard deviation $\sigma[\mathrm{O} / \mathrm{Fe}]_{7774}=0.039$, and

$[\mathrm{O} / \mathrm{Fe}]_{6300}=-0.006( \pm 0.009)-0.533( \pm 0.045)[\mathrm{Fe} / \mathrm{H}]$

with $\sigma[\mathrm{O} / \mathrm{Fe}]_{6300}=0.047$.
For $[\mathrm{O} / \mathrm{Fe}]_{7774}$ and $[\mathrm{O} / \mathrm{Fe}]_{6300}$, the standard deviations agree well with the errors estimated in Table 6 , but in the case of $[\mathrm{C} / \mathrm{Fe}]$ the standard deviation of the regression $( \pm 0.047 \mathrm{dex})$ is higher that the estimated error $( \pm 0.028 \mathrm{dex})$. We note, in this connection, that an extension of the linear regression to include terms in $T_{\text {eff }}$ and $\log g$ does not decrease the standard deviation significantly (from \pm 0.047 dex to \pm 0.044 dex).

As seen from Figs. 14 and 15, stars with metallicities close to that of the Sun tend to fall below the fits suggesting that linear regression is not providing the right mean values of $[\mathrm{C} / \mathrm{Fe}]$ and $[\mathrm{O} / \mathrm{Fe}]$ for stars with $[\mathrm{Fe} / \mathrm{H}] \simeq 0.0$. For the group of 11 stars with $-0.1<[\mathrm{Fe} / \mathrm{H}]<+0.1^{7}$, the average values are $\langle[\mathrm{C} / \mathrm{Fe}]\rangle=$ $-0.052,\left\langle[\mathrm{O} / \mathrm{Fe}]_{7774}\right\rangle=-0.029$, and $\left\langle[\mathrm{O} / \mathrm{Fe}]_{6300}\right\rangle=-0.016$. The small offsets of $[\mathrm{O} / \mathrm{Fe}]$ may be explained as due to accidental errors in the measured equivalent widths of the O I triplet and [O I] $\lambda 6300$ lines in the solar spectrum, but to explain the offset in $[\mathrm{C} / \mathrm{Fe}]$ in this way, the solar EWs of the $\mathrm{C}$ I lines at $5052 \AA$ and $5380 \AA$ would have to be overestimated by more than $2 \mathrm{~m} \AA$. This is unlikely to be the case, because the HARPS spectrum of the Sun was obtained from reflected sunlight of Ceres and Ganymede in the same way as the stellar spectra. The spectra of these minor planets have $\mathrm{S} / \mathrm{N}$ of 350 and 250, respectively, and the EWs in the two spectra agree within $0.7 \mathrm{~m} \AA$ for the $\lambda 5052$ line and within $0.2 \mathrm{~m} \AA$ for the $\lambda 5380$ line. We also note that the stellar $T_{\text {eff }}$-scale at $[\mathrm{Fe} / \mathrm{H}] \simeq 0$ has to be wrong by $\sim 150 \mathrm{~K}$ to explain the offset of $[\mathrm{C} / \mathrm{Fe}]$, which is much larger than the error of $\pm 15 \mathrm{~K}$ (estimated from solar twin stars) of the Cassagrande et al. (2010) IRFM calibration. Hence, it seems that the difference of $[\mathrm{C} / \mathrm{Fe}]$ between the Sun and stars with solar metallicity is real. Furthermore, we note that stars without detected planets in Fig. 14 tend to fall below stars proven to have planets. Fitting the two samples separately, we get

$[\mathrm{C} / \mathrm{Fe}]=-0.003( \pm 0.011)-0.215( \pm 0.047)[\mathrm{Fe} / \mathrm{H}]$

for 22 stars with planets, and

$[\mathrm{C} / \mathrm{Fe}]=-0.051( \pm 0.011)-0.132( \pm 0.059)[\mathrm{Fe} / \mathrm{H}]$

for 18 stars without planets. The difference in zero-points is significant at the 3-sigma level, and it is intriguing that stars with planets have the same $[\mathrm{C} / \mathrm{Fe}]$ at $[\mathrm{Fe} / \mathrm{H}] \simeq 0.0$ as the Sun.

It is tempting to think that the scatter in $[\mathrm{C} / \mathrm{Fe}]$ and the possible systematic difference in $[\mathrm{C} / \mathrm{Fe}]$ between stars with and without planets are related to the variations in the volatile-torefractory ratio found in the works of Meléndez et al. (2009) and Ramírez et al. (2014). However, similar "cosmic" variations are not seen for $[\mathrm{O} / \mathrm{Fe}]$; there is no significant difference in the trends of $[\mathrm{O} / \mathrm{Fe}]$ for stars with and without planets, and the scatter around the fitted lines is not larger than expected from the estimated error of $[\mathrm{O} / \mathrm{Fe}]$. This seems to rule out that the variations in $[\mathrm{C} / \mathrm{Fe}]$ (if real) can be explained as due to variations in the volatile-to-refractory ratio.

\section{Conclusions}

In this paper, we have determined precise $\mathrm{C}$ and $\mathrm{O}$ abundances for $\mathrm{F}$ and $\mathrm{G}$ main-sequence stars in the solar neighborhood belonging to four different populations defined from their sequences in the $[\alpha / \mathrm{Fe}]-[\mathrm{Fe} / \mathrm{H}]$ diagram: thin- and thick-disk stars, high- and low-alpha halo stars. Based on the

7 The average values of the atmospheric parameters of these stars, $\left\langle T_{\text {eff }}\right\rangle=5808 \mathrm{~K},\langle\log g\rangle=4.43$, and $\langle[\mathrm{Fe} / \mathrm{H}]\rangle=0.005$, are close to the solar values. 
trends of $[\mathrm{C} / \mathrm{Fe}],[\mathrm{O} / \mathrm{Fe}]$, and $[\mathrm{C} / \mathrm{O}]$, we make the following conclusions.

i) The high- and low-alpha halo populations are clearly separated in $[\mathrm{C} / \mathrm{Fe}]$ and $[\mathrm{O} / \mathrm{Fe}]$ for the metallicity range $-1.6<$ $[\mathrm{Fe} / \mathrm{H}]<-0.8$ (see Figs. 9 and 10). The high-alpha stars have approximately constant values of $[\mathrm{C} / \mathrm{Fe}] \simeq 0.2$ and $[\mathrm{O} / \mathrm{Fe}] \simeq 0.6$, whereas the low-alpha stars show decreasing trends in $[\mathrm{C} / \mathrm{Fe}]$ and $[\mathrm{O} / \mathrm{Fe}]$ as a function of increasing metallicity and present a higher dispersion in these abundance ratios than the high-alpha stars. These chemical properties and the kinematics of the stars can be explained in a scenario, where the high-alpha stars formed in situ in a dissipative collapse of proto-Galactic gas clouds, where mainly massive stars exploding as Type II SNe contributed to the chemical enrichment. The low-alpha stars, on the other hand, may have been accreted from dwarf galaxies with a relatively slow and bursting star formation history, so that Type Ia SNe started to contribute iron at $[\mathrm{Fe} / \mathrm{H}] \simeq-1.6$.

ii) For metallicities below $[\mathrm{Fe} / \mathrm{H}] \simeq-0.4$, stars with thick-disk kinematics follow the same trends in $[\mathrm{C} / \mathrm{Fe}]$ and $[\mathrm{O} / \mathrm{Fe}]$ as the high-alpha halo stars. In both populations, $[\mathrm{O} / \mathrm{Fe}]$ begins to decline at $[\mathrm{Fe} / \mathrm{H}] \simeq-0.6$ because Type Ia SNe start to contribute iron at this metallicity. This suggest a similar origin of thick-disk and high-alpha halo stars, possibly during the dissipative collaps of proto-Galactic gas clouds.

iii) Although only a few metal-rich thick-disk stars are included in this study, we find clear evidence that thin- and thick disk stars (classified from $[\alpha / \mathrm{Fe}]$ ) are separated in $[\mathrm{C} / \mathrm{Fe}]$ and $[\mathrm{O} / \mathrm{Fe}]$ in the metallicity range $-0.6<[\mathrm{Fe} / \mathrm{H}]<+0.1$. This supports recent evidence of the existence of alpha-enhanced, metal-rich stars (Adibekyan et al. 2012; Bensby et al. 2014) that are older than the thin-disk stars (Haywood et al. 2013).

iv) The $[\mathrm{C} / \mathrm{O}]-[\mathrm{O} / \mathrm{H}]$ diagram (Fig. 12) shows no offset in $[\mathrm{C} / \mathrm{O}]$ between the high- and low-alpha halo stars, which suggests that mainly high-mass $\left(M>8 M_{\odot}\right)$ stars have contributed $\mathrm{C}$ and $\mathrm{O}$ in these populations. The rise in $[\mathrm{C} / \mathrm{O}]$ at $[\mathrm{O} / \mathrm{H}]>-0.4$ for the high-alpha and thick-disk populations may be due to a metallicity dependent carbon yield of such high-mass stars (Cescutti et al. 2009) and/or the C contribution from low-mass stars (Carigi et al. 2005). The thin-disk stars show an offset in $[\mathrm{C} / \mathrm{O}]$ relative to the thick-disk stars due to additional C contribution from low-mass AGB star and massive stars of high metallicity (Carigi et al. 2005; Carigi \& Peimbert 2011), which is made possible due to the younger age of the thin-disk population.

v) The C/O ratio of thin-disk stars (Fig. 13) shows a tight, increasing trend as a function of $[\mathrm{Fe} / \mathrm{H}]$, but even at the highest metallicities, $[\mathrm{Fe} / \mathrm{H}] \simeq+0.4$, the ratio does not exceed 0.8 , i.e., the critical value in a proto-planetary disk for the formation of carbon planets according to Bond et al. (2010). This confirms recent results obtained by Nissen (2013) and Teske et al. (2014). There is some evidence of higher $\mathrm{C} / \mathrm{O}$ ratios in stars hosting planets than in stars without detected planets, but this needs to be confirmed.

vi) In a sample of solar analog stars, there is evidence of a small $(0.05 \pm 0.016 \mathrm{dex})$ systematic difference in $[\mathrm{C} / \mathrm{Fe}]$ between stars with and without detected planets. An attempt to explain this in terms of variations in the volatile-to-refractory element ratio (Meléndez et al. 2009: Ramírez et al. 2014) fails, because a similar difference in $[\mathrm{O} / \mathrm{Fe}]$ between stars with and without planets is not found.

Acknowledgements. This project is supported by the National Natural Science Foundation of China through Grant No. 11390371. P.E.N. acknowledges a visiting professorship at the National Astronomical Observatories in Beijing granted by the Chinese Academy of Sciences (Contract no. 6-1309001). L.C. thanks for the financial supports provided by CONACyT of Mexico (grant 129753) and by MINECO of Spain (AYA2010-16717 and AYA2011-22614). Funding for the Stellar Astrophysics Centre is provided by the Danish National Research Foundation (Grant agreement no.: DNRF106). The research is supported by the ASTERISK project (ASTERoseismic Investigations with SONG and Kepler) funded by the European Research Council (Grant agreement no.: 267864). This research made use of the SIMBAD database operated at CDS, Strasbourg, France, the Exoplanet Orbit Database and the Exoplanet Data Explorer at http://exoplanets.org, and of data products from the Two Micron All Sky Survey, which is a joint project of the University of Massachusetts and the Infrared Processing and Analysis Center/California Institute of Technology, funded by NASA and the National Science Foundation.

\section{References}

Adibekyan, V. Zh., Santos, N. C., Sousa, S. G., \& Israelian, G. 2011, A\&A, 535, L11

Adibekyan, V. Zh., Sousa, S. G. , Santos, N. C., et al. 2012, A\&A, 545, A32

Adibekyan, V. Zh., Figueira, P., Santos, N. C., et al. 2013, A\&A, 554, A44

Akerman, C. J., Carigi, L., Nissen, P. E., Pettini, M., \& Asplund, M. 2004, A\&A, 414,931

Allende Prieto, C., Lambert, D. L., \& Asplund, M. 2001, ApJ, 556, L63

Asplund, M. 2005, ARA\&A, 43, 481

Asplund, M., Grevesse, N., Sauval, A. J., \& Scott, P. 2009, ARA\&A, 47, 481

Barklem, P. S. 2007, A\&A, 462, 781

Barklem, P. S., \& Aspelund-Johansson, J. 2005, A\&A, 435, 373

Barklem, P. S., Piskunov, N., \& O’Mara, B. J. 2000, A\&AS, 142, 467

Bensby, T., \& Feltzing, S. 2006, MNRAS, 367, 1181

Bensby, T., Feltzing, S., \& Lundström, I. 2004, A\&A, 415, 155

Bensby, T., Feltzing, S., Lundström, I., \& Ilyin, I. 2005, A\&A, 433, 185

Bensby, T., Feltzing, S., \& Oey, M. S. 2014, A\&A, 562, A71

Bond, J. C., O'Brien, D. P., \& Lauretta, D. S. 2010, ApJ, 715, 1050

Bond, H. E., Nelan, E. P., VandenBerg, D. A., Schaefer, G. H., \& Harmer, D. 2013, ApJ, 765, L12

Caffau, E., Ludwig, H.-G., Steffen, M., et al. 2008, A\&A, 488, 1031

Caffau, E., Ludwig, H.-G., Bonifacio, P., et al. 2010, A\&A, 514, A92

Caffau, E., Ludwig, H.-G., Malherbe, J.-M., et al. 2013, A\&A, 554, A126

Carigi, L., \& Hernandez, X. 2008, MNRAS, 390, 582

Carigi, L., \& Peimbert, M. 2011, RMxAA, 47, 139

Carigi, L., Hernandez, X., \& Gilmore, G. 2002, MNRAS, 334, 117

Carigi, L., Peimbert, M., Esteban, C., \& García-Rojas, J. 2005, ApJ, 623, 213

Carretta, E., Bragaglia, A., Gratton, R., \& Lucatello, S. 2009, A\&A, 505, 139

Casagrande, L., Ramírez. I., Meléndez, J., Bessell, M., \& Asplund, M. 2010, A\&A, 512, A54

Cayrel, R., Depagne, E., Spite, M., et al. 2004, A\&A, 416, 1117

Cescutti, G., Matteucci, F., McWilliam, A., \& Chiappini, C. 2009, A\&A, 505, 605

Chiappini, C., Matteucci, F., \& Meynet, G. 2003, A\&A, 410, 257

Delgado Mena, E., Israelian, G., González Hernández, J. I., et al. 2010, ApJ, 725, 2349

Drawin, H.-W. 1968, Z. für Phys., 211, 404

Fabbian, D., Asplund, M., Barklem, P. S., Carlsson, M., \& Kiselman, D. 2009, A\&A, 500, 1221

Fortney, J. J. 2012, ApJ, 747, L27

Fulbright, J. P., \& Johnson, J. A. 2003, ApJ, 595, 1154

García Pérez, A. E., Asplund, M., Primas, F., Nissen, P. E., \& Gustafsson, B. 2006, A\&A, 451, 621

González Hernández, J. I., Israelian, G., Santos, N. C., et al. 2010, ApJ, 720, 1592

González Hernández, J. I., Delgado-Mena, E., Sousa, S. G., et al. 2013, A\&A, 552, A6

Gustafsson, B., Karlsson, T., Olsson, E., Edvardsson, B., \& Ryde, N. 1999, A\&A, 342, 426

Gustafsson, B., Edvardsson, B., Eriksson, K., et al. 2008, A\&A, 486, 951

Haywood, M., Di Matteo, P., Lehnert, M. D., Katz, D., \& Gómez, A. 2013, A\&A, 560, A109

Hibbert, A., Biémont, E., Godefroid, M., \& Vaeck, N. 1991, J. Phys. B, 24, 3943 Hibbert, A., Biémont, E., Godefroid, M., \& Vaeck, N. 1993, A\&AS, 99, 179

Johansson, S., Litzén, U., Lundberg, H., \& Zhang, Z. 2003, ApJ, 584, L107

Kaufer, A., Stahl, O., Tubbesing, K., et al. 1999, The Messenger, 95, 8

Kiselman, D. 1993, A\&A, 275, 269

Kobayashi, C., Umeda, H., Nomoto, K., Tominaga, N., \& Ohkubo, T. 2006, ApJ, 653, 1145

Kobayashi, C., Karakas, A. I., \& Umeda, H. 2011, MNRAS, 414, 3231

Kobayashi, C., Ishigaki, M. N., Tominaga, N., \& Nomoto, K. 2014 ApJ, 785, L5 
P. E. Nissen et al.: Carbon and oxygen abundances in stellar populations

Kuchner, M. J., \& Seager, S. 2005 [arXiv: astro-ph/0504214] Lind, K., Bergemann, M., \& Asplund, M. 2012, MNRAS, 427, 50

Liu, S., Nissen, P. E., Schuster, W. J., et al. 2012, A\&A, 541, A48

Lodders, K. 2003, ApJ, 591, 1220

Madhusudhan, N., Lee, K. K. M., \& Mousis, O. 2012, ApJ, 759, L40

Mashonkina, L., Gehren, T., Shi, J.-R, Korn, A. J., \& Grupp, F. 2011, A\&A, 528, A87

Mayor, M., Pepe, F., Queloz, D., et al. 2003, The Messenger , 114, 20

Meléndez, J., \& Barbuy, B. 2009, A\&A, 497, 611

Meléndez, J., Asplund, M., Gustafsson, B., \& Yong, D. 2009, ApJ, 704, L66

Meynet, G., \& Maeder, A. 2002, A\&A, 390, 56

Nissen, P. E. 2013, A\&A, 552, A73

Nissen, P. E., \& Schuster, W. J. 1997, A\&A, 326, 751

Nissen, P. E., \& Schuster, W. J. 2010, A\&A, 511, L10

Nissen, P. E., \& Schuster, W. J. 2011, A\&A, 530, A15

Nissen, P. E., \& Schuster, W. J. 2012, A\&A, 543, A28

Nissen, P. E., \& Schuster, W. J. 2014, in Setting the scene for GAIA and LAMOST, eds. S. Feltzing, G. Zhao, N. A. Walton, \& P. A. Whitelock (Cambridge Univ. Press), Proc. IAU Symp., 298, 65

Nissen, P. E., Primas, F., Asplund, M., \& Lambert, D. L. 2002, A\&A, 390, 235

Olsen, E. H. 1983, A\&AS, 54, 55

Pereira, T. M. D., Asplund, M., \& Kiselman, D. 2009, A\&A, 508, 1403

Perryman, M. A. C. (ed.) 1997, The HIPPARCOS and TYCHO catalogues, ESA SP, 1200

Petigura, E. A., \& Marcy, G. W. 2011, ApJ, 735, 41

Ramírez, I., \& Meléndez, J. 2005, ApJ, 626, 465

Ramírez, I., Meléndez, J., \& Chanamé, J. 2012, ApJ, 757, 164
Ramírez, I., Allende Prieto, C., \& Lambert, D. L. 2013, ApJ., 764, 78

Ramírez, I., Meléndez, J., \& Asplund, M. 2014, A\&A, 561, A7

Reddy, B. E., Lambert, D. L., \& Allende Prieto, C. 2006, MNRAS, 367, 1329

Schuler, S. C., King, J. R., Hobbs, L. M., \& Pinsonneault, M. H. 2004, ApJ, 602, L117

Schuler, S. C., King, J. R., Terndrup, D. M., et al. 2006, ApJ, 636, 432

Schuster, W. J., Moreno, E., Nissen, P. E., \& Pichardo, B. 2012, A\&A, 538, A21

Scott, P., Asplund, M., Grevesse, N., \& Sauval, J. 2009, ApJ, 691, L119

Shi, J. R., Zhao, G., \& Chen, Y. Q. 2002, A\&A, 381, 982

Skrutskie, M. F., Cutri, R. M., Stiening, R., et al. 2006, AJ, 131, 1163

Sousa, S. G., Santos, N. C., Mayor, M., et al. 2008, A\&A, 487, 373

Sousa, S. G., Santos, N. C., Israelian, G., et al. 2011a, A\&A, 526, A99

Sousa, S. G., Santos, N. C., Israelian, G., Mayor, M., \& Udry, S. 2011b, A\&A 533, A141

Takeda, Y., \& Honda, S. 2005, PASJ, 57, 65

Takeda, Y., \& Takada-Hidai, M. 2013, PASJ, 65, 65

Teske, J. K., Cunha, K., Schuler, S. C., Griffith, C. A., \& Smith, V. V. 2013, ApJ, 778,132

Teske, J. K., Cunha, K., Smith, V. V., Schuler, S. C., \& Griffith, C. A. 2014, ApJ, 788,39

Tolstoy, E., Hill, V., \& Tosi, M. 2009, ARA\&A, 47, 37

Unsöld, A. 1955, Physik der Sternatmosphären, 2nd ed. (Berlin: Springer Verlag) van den Hoek, L. B., \& Groenewegen, M. A. T. 1997, A\&AS, 123, 305

van Leeuwen, F. 2007, HIPPARCos, the New Reduction of the Raw Data, Astrophys. Space Sci. Library (Dordrecht: Springer), 350

Wright, J. T., Fakhouri, O., Marcy, G. W., et al. 2011, PASP, 123, 412

Yi, S. K., Kim, Y. -C., \& Demarque, P. 2003, ApJS, 144, 259 
A\&A 568, A25 (2014)

Table 2. ESO observing program numbers, $\mathrm{S} / \mathrm{N}$ of spectra, and measured equivalent widths for the HARPS-FEROS sample.

\begin{tabular}{|c|c|c|c|c|c|c|c|c|c|c|c|}
\hline \multirow[b]{2}{*}{ ID } & \multicolumn{2}{|c|}{ HARPS } & \multicolumn{2}{|c|}{ FEROS } & \multicolumn{7}{|c|}{$E W(\mathrm{~m} \AA)$} \\
\hline & Program & $S / N$ & Program & $S / N$ & $\mathrm{CI}_{5052}$ & $\mathrm{CI}_{5380}$ & $\mathrm{OI}_{7772}$ & $\mathrm{O}_{7774}$ & O I 7775 & {$[\mathrm{O} \mathrm{I}]+\mathrm{Ni}^{a}$} & {$[\mathrm{O} \mathrm{I}]^{b}$} \\
\hline HD 1237 & 72.C-0488 & 350 & 60.A-9700 & 200 & 30.9 & 16.8 & 59.1 & 50.2 & 36.4 & 6.0 & 3.5 \\
\hline HD 4208 & - & 200 & 83.A-9011 & 200 & 21.4 & 11.9 & 52.3 & 42.6 & 33.6 & & \\
\hline HD 4307 & - & 500 & - & 250 & 32.0 & 18.9 & 78.1 & 66.1 & 55.2 & 5.9 & 4.9 \\
\hline HD 4308 & - & 600 & 74.D-0086 & 500 & 25.5 & 14.1 & 68.1 & 58.5 & 42.4 & & \\
\hline HD 14374 & - & 350 & 83.A-9011 & 200 & 22.4 & 11.6 & 43.6 & 38.1 & 31.5 & 5.0 & 3.2 \\
\hline HD 16141 & - & 300 & 83.A-9011 & 250 & 45.2 & 27.9 & 86.3 & 75.7 & 58.6 & & \\
\hline HD 20782 & - & 900 & - & 200 & 33.5 & 19.9 & 72.2 & 59.5 & 49.3 & 5.4 & 4.0 \\
\hline HD 23079 & - & 900 & 84.A-9004 & 200 & 34.1 & 19.7 & 80.8 & 68.5 & 51.8 & 4.2 & 3.2 \\
\hline HD 28185 & - & 500 & 83.A-9011 & 250 & 44.5 & 26.9 & 71.7 & 62.7 & 46.2 & 8.6 & 5.1 \\
\hline HD 30177 & - & 250 & 84.A-9004 & 200 & 48.5 & 31.0 & 72.2 & 62.0 & 53.3 & & \\
\hline HD 30306 & - & 500 & 82.C-0446 & 150 & 38.5 & 21.5 & 63.8 & 54.5 & 45.8 & 7.5 & 4.1 \\
\hline HD 40397 & - & 300 & 78.D-0760 & 200 & 29.4 & 16.7 & 61.0 & 51.3 & 36.4 & 6.4 & 4.6 \\
\hline HD 52265 & - & 250 & 80.A-9021 & 350 & 59.1 & 38.9 & 109.2 & 95.6 & 83.1 & & \\
\hline HD 65216 & - & 500 & 83.A-9003 & 250 & 22.2 & 12.4 & 54.4 & 50.3 & 35.7 & 4.4 & 3.2 \\
\hline HD 65907 & 88.C-0011 & 900 & 78.D-0760 & 150 & 35.2 & 19.6 & 89.2 & 75.2 & 60.5 & 5.2 & 4.5 \\
\hline HD 69830 & 72.C-0488 & 800 & 77.C-0573 & 300 & 23.1 & 12.7 & 42.0 & 37.2 & 28.1 & 5.5 & 3.5 \\
\hline HD 73256 & - & 1100 & 83.A-9003 & 200 & 39.7 & 22.5 & 61.5 & 54.5 & 41.3 & 8.1 & 4.0 \\
\hline HD 75289 & - & 650 & 84.A-9003 & 500 & 57.3 & 36.4 & 108.2 & 95.4 & 78.3 & 5.5 & 3.2 \\
\hline HD 77110 & 82.C-0212 & 500 & 76.B-0416 & 150 & 18.1 & 9.4 & 60.2 & 52.3 & 39.0 & 5.0 & 4.4 \\
\hline HD 78538 & 72.C-0488 & 350 & 77.D-0525 & 150 & 27.7 & 15.5 & 67.2 & 60.9 & 41.6 & 4.4 & 3.0 \\
\hline HD 82943 & - & 700 & 84.A-9004 & 300 & 55.0 & 35.4 & 100.2 & 87.2 & 72.8 & 6.9 & 4.1 \\
\hline HD 89454 & - & 900 & 77.D-0525 & 150 & 35.9 & 20.5 & 68.1 & 65.0 & 49.3 & 5.9 & 3.2 \\
\hline HD 92788 & - & 400 & 80.A-9021 & 250 & 49.0 & 30.0 & 81.1 & 70.1 & 58.3 & 8.5 & 4.6 \\
\hline HD 94151 & - & 400 & 77.D-0525 & 200 & 33.0 & 19.3 & 59.4 & 58.0 & 37.8 & 5.7 & 3.3 \\
\hline HD 96423 & - & 550 & - & 150 & 38.7 & 22.7 & 74.4 & 60.5 & 50.3 & 6.8 & 4.1 \\
\hline HD 102365 & 60.A-9036 & 450 & 88.C-0892 & 250 & 23.0 & 13.5 & 54.3 & 45.6 & 33.8 & 4.6 & 3.6 \\
\hline HD 108147 & 72.C-0488 & 650 & 83.A-9013 & 250 & 55.6 & 35.0 & 114.9 & 98.1 & 80.9 & 4.2 & 2.7 \\
\hline HD 110668 & - & 120 & 77.D-0525 & 150 & 49.0 & 29.7 & 85.5 & 78.6 & 60.6 & & \\
\hline HD 111232 & - & 550 & - & 250 & 19.6 & 10.5 & 54.8 & 45.5 & 33.0 & & \\
\hline HD 114613 & - & 700 & 80.D-2002 & 250 & 50.3 & 30.7 & 83.5 & 76.6 & 63.1 & 9.7 & 6.7 \\
\hline HD 114729 & - & 900 & - & 350 & 31.6 & 18.2 & 76.0 & 64.9 & 52.6 & 6.2 & 5.3 \\
\hline HD 114853 & - & 750 & 77.D-0525 & 150 & 22.4 & 12.8 & 58.0 & 47.2 & 36.2 & & \\
\hline HD 115617 & 72.D-0707 & 450 & 86.D-0460 & 150 & 27.3 & 15.7 & 52.7 & 48.7 & 31.4 & 6.1 & 4.1 \\
\hline HD 117207 & 72.C-0488 & 200 & 83.A-9013 & 200 & 43.8 & 26.5 & 74.3 & 61.1 & 51.5 & & \\
\hline HD 117618 & - & 600 & - & 300 & 44.8 & 28.1 & 92.1 & 79.4 & 60.2 & 5.0 & 3.4 \\
\hline HD 125184 & - & 850 & 85.C-0557 & 250 & 50.5 & 32.9 & 79.6 & 73.0 & 58.7 & 9.4 & 5.0 \\
\hline HD 125612 & - & 350 & 77.D-0525 & 200 & 45.1 & 27.7 & 88.8 & 76.0 & 62.0 & 7.2 & 4.4 \\
\hline HD 126525 & - & 500 & - & 150 & 29.8 & 16.8 & 59.2 & 52.9 & 35.4 & 5.7 & 4.1 \\
\hline HD 128674 & - & 500 & 76.B-0416 & 150 & 17.2 & 8.8 & 45.5 & 39.4 & 24.3 & 3.6 & 2.7 \\
\hline HD 134664 & - & 600 & 77.D-0525 & 150 & 37.6 & 21.8 & 75.3 & 70.5 & 48.8 & 5.5 & 3.7 \\
\hline HD 134987 & - & 550 & 83.A-9013 & 250 & 53.7 & 33.8 & 87.5 & 76.4 & 57.8 & 8.9 & 4.8 \\
\hline HD 136352 & - & 700 & 89.C-0440 & 350 & 26.2 & 15.1 & 65.5 & 56.9 & 43.2 & 5.8 & 5.0 \\
\hline HD 140901 & - & 650 & 78.A-9059 & 200 & 33.2 & 19.2 & 60.1 & 53.5 & 42.8 & 5.7 & 3.1 \\
\hline HD 145666 & - & 550 & 77.D-0525 & 150 & 34.3 & 20.4 & 80.6 & 67.6 & 57.3 & 4.4 & 3.1 \\
\hline HD 146233 & - & 1000 & 78.A-9007 & 300 & 35.8 & 21.2 & 73.1 & 62.2 & 51.3 & 5.5 & 3.6 \\
\hline HD 157347 & - & 450 & 89.C-0440 & 500 & 32.0 & 19.1 & 66.1 & 56.9 & 44.3 & 6.2 & 4.2 \\
\hline HD 160691 & 73.D-0578 & 600 & 83.A-9013 & 250 & 54.1 & 35.1 & 89.2 & 77.1 & 65.6 & 9.5 & 5.5 \\
\hline HD 168443 & 72.C-0488 & 600 & 83.A-9003 & 300 & 41.6 & 25.5 & 72.9 & 65.5 & 50.7 & 9.8 & 7.1 \\
\hline HD 169830 & - & 550 & 83.A-9013 & 350 & 70.5 & 46.9 & 139.3 & 123.8 & 102.5 & 6.2 & 4.8 \\
\hline HD 179949 & - & 450 & 85.C-0743 & 450 & 59.7 & 38.2 & 118.5 & 101.9 & 83.4 & & \\
\hline HD 183263 & 75.C-0332 & 500 & 79.A-9013 & 250 & 57.4 & 37.5 & 98.7 & 84.4 & 67.7 & & \\
\hline HD 190248 & 74.D-0380 & 700 & 77.A-9009 & 150 & 52.7 & 31.3 & 74.8 & 68.0 & 56.3 & 10.1 & 4.6 \\
\hline HD 196050 & 72.C-0488 & 950 & 83.A-9011 & 300 & 56.8 & 37.1 & 97.5 & 82.6 & 69.5 & 8.2 & 5.0 \\
\hline HD 196761 & - & 750 & 77.A-9009 & 200 & 17.5 & 8.9 & 36.2 & 30.0 & 21.9 & & \\
\hline
\end{tabular}

Notes. ${ }^{(a)}$ The EW of the [O I] + Ni I blend at $6300.3 \AA .{ }^{(b)}$ The EW of [O I] $\lambda 6300.3$ after correcting for the contribution of the Ni I line. 
P. E. Nissen et al.: Carbon and oxygen abundances in stellar populations

Table 2. continued.

\begin{tabular}{|c|c|c|c|c|c|c|c|c|c|c|c|}
\hline \multirow[b]{2}{*}{ ID } & \multicolumn{2}{|c|}{ HARPS } & \multicolumn{2}{|c|}{ FEROS } & \multirow[b]{2}{*}{$\mathrm{CI}_{5052}$} & \multirow[b]{2}{*}{$\mathrm{CI}_{5380}$} & \multicolumn{3}{|c|}{ 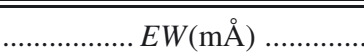 } & \multirow[b]{2}{*}[\mathrm{OI}]{$+\mathrm{Ni}^{a}$} & \multirow[b]{2}{*}[\mathrm{OI}]{$^{b}$} \\
\hline & Program & $S / N$ & Program & $S / N$ & & & O I 7772 & O I 7774 & O I 7775 & & \\
\hline HD 202206 & - & 700 & 83.A-9011 & 200 & 43.3 & 26.7 & 74.7 & 67.0 & 48.7 & 6.8 & 3.3 \\
\hline HD 203608 & 77.D-0720 & 600 & 85.A-9027 & 300 & 19.7 & 11.0 & 70.5 & 61.2 & 48.5 & 2.0 & 1.8 \\
\hline HD 206172 & 72.C-0488 & 200 & 77.C-0192 & 120 & 20.4 & 11.3 & 53.8 & 50.4 & 34.0 & & \\
\hline HD 207129 & - & 800 & 60.A-9122 & 300 & 35.7 & 20.7 & 80.7 & 67.4 & 54.0 & 5.0 & 3.6 \\
\hline HD 210277 & - & 900 & - & 200 & 41.1 & 25.0 & 65.1 & 57.7 & 44.4 & 8.5 & 4.8 \\
\hline HD 212301 & 82.C-0312 & 350 & 85.C-0743 & 350 & 58.4 & 37.2 & 112.8 & 97.6 & 81.8 & & \\
\hline HD 213240 & 72.C-0488 & 300 & 83.A-9011 & 200 & 51.9 & 33.5 & 99.1 & 82.5 & 71.1 & 6.9 & 4.7 \\
\hline HD 216435 & - & 700 & - & 200 & 61.2 & 40.1 & 108.9 & 96.5 & 77.1 & 7.7 & 4.8 \\
\hline HD 216777 & - & 330 & 77.C-0192 & 120 & 17.2 & 8.9 & 46.5 & 39.3 & 32.9 & & \\
\hline HD 216437 & 80.D-0408 & 700 & - & 200 & 36.7 & 21.6 & 52.4 & 47.3 & 37.3 & 8.9 & 5.5 \\
\hline HD 216770 & 72.C-0488 & 600 & - & 120 & 54.9 & 36.9 & 96.3 & 81.8 & 65.7 & 8.5 & 3.5 \\
\hline HD 222669 & - & 650 & 77.D-0525 & 150 & 39.5 & 23.6 & 79.0 & 71.9 & 55.2 & 5.2 & 3.4 \\
\hline
\end{tabular}

Table 3. Equivalent widths for the UVES-FIES sample of halo and thick-disk stars.

\begin{tabular}{|c|c|c|c|c|c|c|c|c|c|c|}
\hline \multirow[b]{2}{*}{ ID } & \multicolumn{2}{|c|}{$\ldots . . E W(\mathrm{~m} \AA) \ldots .}$. & \multirow[b]{2}{*}{ ref. $^{a}$} & \multicolumn{3}{|c|}{$E W(\mathrm{~m} \AA)$} & \multirow[b]{2}{*}{ ref. $^{b}$} & \multicolumn{2}{|c|}{$E W(\mathrm{~m} \AA) \ldots .}$. & \multirow[b]{2}{*}{ ref. $^{e}$} \\
\hline & $\mathrm{CI}_{5052}$ & $\mathrm{CI}_{5380}$ & & O I 7772 & O I 7774 & O I 7775 & & {$[\mathrm{OI}]+\mathrm{Ni}^{c}$} & {$[\mathrm{O} \mathrm{I}]^{d}$} & \\
\hline BD-21 3420 & 7.4 & 4.1 & $\mathrm{U}$ & 49.4 & 43.4 & 34.6 & $\mathrm{R}, \mathrm{E}$ & 2.2 & 2.1 & $\mathrm{U}$ \\
\hline CD-33 3337 & 7.2 & 3.7 & $\mathrm{U}$ & 51.5 & 42.6 & 31.6 & $\mathrm{R}, \mathrm{E}$ & & & \\
\hline CD-43 6810 & 33.7 & 18.6 & $\mathrm{U}$ & 94.7 & 81.6 & 65.0 & $\mathrm{R}$ & 5.1 & 4.6 & $\mathrm{U}$ \\
\hline CD-45 3283 & 3.2 & 2.0 & $\mathrm{U}$ & 32.2 & 26.1 & 20.1 & $\mathrm{R}, \mathrm{E}$ & & & \\
\hline CD-51 4628 & 3.3 & 2.4 & $\mathrm{U}$ & 41.5 & 33.2 & 23.8 & $\mathrm{R}$ & & & \\
\hline CD-57 1633 & 7.9 & 3.8 & $\mathrm{U}, \mathrm{H}$ & 47.1 & 40.2 & 29.7 & $\mathrm{R}, \mathrm{E}$ & & & \\
\hline CD-61 0282 & 3.0 & 1.5 & $\mathrm{U}$ & 35.8 & 28.9 & 22.1 & $\mathrm{R}, \mathrm{E}$ & & & \\
\hline G05-19 & 3.7 & 2.1 & $\mathrm{U}$ & 40.1 & 33.1 & 23.1 & $\mathrm{R}$ & & & \\
\hline G05-36 & 6.8 & & $\mathrm{~F}$ & 52.0 & 45.0 & 39.0 & $\mathrm{R}$ & & & \\
\hline G05-40 & 15.0 & 8.0 & $\mathrm{U}$ & 66.1 & 57.5 & 44.0 & $\mathrm{R}, \mathrm{E}$ & & & \\
\hline G15-23 & 3.0 & & $\mathrm{~F}$ & 21.9 & 18.8 & 13.5 & $\mathrm{R}$ & & & \\
\hline G18-28 & 7.3 & & $\mathrm{U}$ & 34.9 & & 22.2 & $\mathrm{R}$ & & & \\
\hline G18-39 & 4.8 & 2.1 & $\mathrm{U}$ & 41.8 & 36.9 & 25.7 & $\mathrm{U}$ & & & \\
\hline G20-15 & 3.0 & 1.5 & $\mathrm{U}$ & 30.4 & 27.7 & 16.9 & $\mathrm{R}$ & & & \\
\hline G21-22 & 6.2 & 1.9 & $\mathrm{~F}$ & & & & & & & \\
\hline G24-13 & 11.8 & 6.6 & $\mathrm{~F}$ & 62.8 & 56.5 & 42.0 & $\mathrm{R}$ & & & \\
\hline G31-55 & 5.2 & 3.2 & $\mathrm{~F}$ & 40.4 & 32.8 & 22.3 & $\mathrm{R}$ & & & \\
\hline G46-31 & 12.3 & 6.3 & $\mathrm{U}$ & 58.0 & 50.3 & 36.9 & $\mathrm{E}$ & 2.5 & 2.3 & $\mathrm{U}$ \\
\hline G49-19 & 23.0 & 11.5 & $\mathrm{~F}$ & & & & & & & \\
\hline G53-41 & 2.6 & 1.6 & $\mathrm{~F}$ & & 21.8 & 14.1 & $\mathrm{R}$ & & & \\
\hline G56-30 & 6.4 & 3.3 & $\mathrm{~F}$ & & & & & & & \\
\hline G56-36 & 10.4 & 4.8 & $\mathrm{~F}$ & 58.6 & 50.0 & 38.5 & $\mathrm{R}$ & & & \\
\hline G57-07 & 20.8 & 11.6 & $\mathrm{~F}$ & & & & & & & \\
\hline G63-26 & 4.5 & 1.9 & $\mathrm{U}$ & & & & & & & \\
\hline G66-22 & 2.3 & 1.3 & $\mathrm{U}$ & 20.0 & 18.6 & 10.1 & $\mathrm{R}$ & & & \\
\hline G74-32 & 15.3 & 7.7 & $\mathrm{~F}$ & & & & & & & \\
\hline G75-31 & 7.2 & & $\mathrm{~F}$ & 55.7 & 44.4 & 34.3 & $\mathrm{U}$ & & & \\
\hline G81-02 & 18.3 & 9.2 & $\mathrm{~F}$ & & & & & & & \\
\hline G82-05 & 5.4 & 2.3 & $\mathrm{U}$ & 24.0 & 20.0 & 13.0 & $\mathrm{R}$ & & & \\
\hline G85-13 & 13.7 & 7.8 & $\mathrm{~F}$ & 59.0 & 49.1 & 39.5 & $\mathrm{R}$ & & & \\
\hline G87-13 & 6.4 & 1.9 & $\mathrm{~F}$ & & & & & & & \\
\hline G96-20 & 20.9 & 11.5 & $\mathrm{~F}$ & & & & & & & \\
\hline G98-53 & 9.9 & 5.3 & $\mathrm{~F}$ & & & & & & & \\
\hline G99-21 & 12.7 & 6.9 & $\mathrm{~F}$ & 50.7 & 39.1 & 31.8 & $\mathrm{R}$ & & & \\
\hline G112-43 & 6.7 & 3.3 & $\mathrm{U}$ & 47.2 & 40.0 & 28.0 & $\mathrm{R}$ & & & \\
\hline
\end{tabular}

Notes. ${ }^{(a)}$ Reference for EWs of C I lines: U, UVES; F, FIES; H, HARPS. ${ }^{(b)}$ Reference for EWs of O I lines: R, Ramirez et al. (2012); E, EMMI; U, UVES; F, FEROS. ${ }^{(c)}$ The EW of the [OI] + Ni I blend at $6300.3 \AA .{ }^{(d)}$ The EW of [OI] $\lambda 6300.3$ after correcting for the contribution of the Ni I line. ${ }^{(e)}$ Reference for EWs of the [O I] line: U, UVES; H, HARPS. 
Table 3. continued.

\begin{tabular}{|c|c|c|c|c|c|c|c|c|c|c|}
\hline \multirow[b]{2}{*}{ ID } & \multicolumn{2}{|c|}{$\ldots . E W(\mathrm{~m} \AA) \ldots}$. & \multirow[b]{2}{*}{ ref. $^{a}$} & \multicolumn{3}{|c|}{$E W(\mathrm{~m} \AA)$} & \multirow[b]{2}{*}{ ref. $^{b}$} & \multicolumn{2}{|c|}{$\ldots . . E W(\mathrm{~m} \AA)$} & \multirow[b]{2}{*}{ ref. $^{e}$} \\
\hline & $\mathrm{CI}_{5052}$ & $\mathrm{CI}_{5380}$ & & O I 7772 & O I 7774 & O I 7775 & & {$[\mathrm{OI}]+\mathrm{Ni}^{c}$} & {$[\mathrm{O} \mathrm{I}]^{d}$} & \\
\hline G112-44 & 3.5 & 2.4 & $\mathrm{U}$ & & & & & & & \\
\hline G114-42 & 3.2 & 2.0 & $\mathrm{U}$ & & & & & & & \\
\hline G119-64 & 3.5 & & $\mathrm{~F}$ & 37.9 & 34.3 & 22.8 & $\mathrm{R}$ & & & \\
\hline G121-12 & 6.4 & 3.2 & $\mathrm{U}$ & & & & & & & \\
\hline G127-26 & 24.1 & 13.3 & $\mathrm{~F}$ & & & & & & & \\
\hline G150-40 & 6.8 & 4.7 & $\mathrm{~F}$ & 52.8 & 48.0 & 38.0 & $\mathrm{R}$ & & & \\
\hline G159-50 & 8.6 & 4.3 & $\mathrm{U}$ & 41.9 & & 24.7 & $\mathrm{R}$ & & & \\
\hline G161-73 & 7.0 & & $\mathrm{~F}$ & & & & & & & \\
\hline G170-56 & 10.4 & 5.1 & $\mathrm{~F}$ & 58.9 & 50.1 & 36.9 & $\mathrm{R}$ & & & \\
\hline G176-53 & & & & 21.9 & & 10.4 & $\mathrm{R}$ & & & \\
\hline G180-24 & 5.5 & 3.3 & $\mathrm{~F}$ & 44.1 & 38.0 & 29.0 & $\mathrm{R}$ & & & \\
\hline G187-18 & 13.8 & 7.8 & $\mathrm{~F}$ & & & & & & & \\
\hline G188-22 & 6.0 & 2.8 & $\mathrm{U}$ & 53.0 & 43.3 & 33.1 & $\mathrm{R}$ & & & \\
\hline HD 3567 & 6.1 & 3.0 & $\mathrm{U}$ & 49.9 & 41.8 & 31.1 & $\mathrm{R}, \mathrm{U}$ & & & \\
\hline HD 17820 & 19.3 & 10.1 & $\mathrm{U}$ & 71.3 & 61.9 & 45.4 & $\mathrm{R}, \mathrm{E}$ & 4.0 & 3.6 & $\mathrm{U}$ \\
\hline HD 22879 & 13.0 & 7.0 & $\mathrm{U}, \mathrm{H}$ & 62.1 & 53.2 & 41.4 & $\mathrm{R}, \mathrm{F}$ & & & \\
\hline HD 25704 & 13.6 & 7.4 & $\mathrm{U}, \mathrm{H}$ & 62.3 & 49.4 & 37.4 & $\mathrm{R}, \mathrm{E}$ & 2.6 & 2.4 & $\mathrm{U}, \mathrm{H}$ \\
\hline HD 51754 & 20.8 & 11.2 & $\mathrm{U}, \mathrm{H}$ & 70.4 & 62.7 & 44.3 & $\mathrm{R}$ & 3.9 & 3.4 & $\mathrm{U}, \mathrm{H}$ \\
\hline HD 59392 & 3.4 & 1.2 & $\mathrm{U}$ & 37.0 & 28.5 & 19.1 & $\mathrm{R}$ & & & \\
\hline HD 76932 & 13.4 & 7.3 & $\mathrm{U}$ & 66.0 & 56.9 & 44.1 & $\mathrm{R}, \mathrm{U}$ & 3.5 & 3.3 & $\mathrm{U}$ \\
\hline HD 97320 & 9.6 & 4.9 & $\mathrm{U}, \mathrm{H}$ & 55.6 & 45.7 & 35.9 & $\mathrm{R}, \mathrm{F}$ & & & \\
\hline HD 103723 & 11.0 & 5.9 & $\mathrm{U}$ & 57.8 & 49.7 & 37.9 & $\mathrm{U}$ & & & \\
\hline HD 105004 & 10.3 & 5.5 & $\mathrm{U}$ & 45.9 & 37.2 & 28.8 & $\mathrm{U}$ & & & \\
\hline HD 106516 & 24.7 & 12.9 & $\mathrm{U}$ & 98.0 & 84.2 & 66.7 & $\mathrm{R}, \mathrm{E}$ & & & \\
\hline HD 111980 & 8.9 & 5.1 & $\mathrm{U}$ & 62.0 & 51.6 & 38.4 & $\mathrm{R}$ & 2.9 & 2.7 & $\mathrm{U}$ \\
\hline HD 113679 & 17.5 & 9.5 & $\mathrm{U}, \mathrm{H}$ & 69.3 & 60.0 & 44.8 & $\mathrm{R}, \mathrm{E}$ & 4.9 & 4.4 & $\mathrm{U}, \mathrm{H}$ \\
\hline HD 114762A & 18.7 & 10.1 & $\mathrm{U}$ & 70.4 & 60.7 & 46.6 & $\mathrm{R}$ & & & \\
\hline HD 120559 & 6.6 & 3.5 & $\mathrm{U}$ & 31.5 & 29.2 & 20.7 & $\mathrm{R}, \mathrm{E}$ & 3.7 & 3.4 & $\mathrm{U}$ \\
\hline HD 121004 & 13.1 & 7.2 & $\mathrm{U}, \mathrm{H}$ & 57.7 & 47.5 & 35.0 & $\mathrm{R}, \mathrm{E}$ & 3.8 & 3.4 & $\mathrm{U}, \mathrm{H}$ \\
\hline HD 126681 & 3.1 & 2.1 & $\mathrm{U}, \mathrm{H}$ & 29.4 & 24.4 & 16.7 & $\mathrm{R}, \mathrm{E}$ & & & \\
\hline HD 132475 & 3.8 & 1.7 & $\mathrm{U}$ & 38.9 & 29.7 & 21.2 & $\mathrm{R}$ & & & \\
\hline HD 148816 & 19.1 & 10.3 & $\mathrm{U}, \mathrm{H}$ & 73.3 & 62.0 & 46.3 & $\mathrm{R}$ & & & \\
\hline HD 159482 & 14.4 & 7.9 & $\mathrm{~F}$ & 64.7 & 54.9 & 43.6 & $\mathrm{R}$ & & & \\
\hline HD 160693 & 20.7 & 11.1 & $\mathrm{~F}$ & 72.0 & 60.2 & 45.7 & $\mathrm{R}$ & & & \\
\hline HD 163810 & 2.1 & & $\mathrm{U}$ & 20.0 & & 12.2 & $\mathrm{R}$ & & & \\
\hline HD 175179 & 17.6 & 9.6 & $\mathrm{U}$ & 70.3 & 64.3 & 42.3 & $\mathrm{R}$ & 4.3 & 3.9 & $\mathrm{U}$ \\
\hline HD 179626 & 9.5 & 5.4 & $\mathrm{U}$ & 59.0 & 50.2 & 39.6 & $\mathrm{R}$ & & & \\
\hline HD 189558 & 6.6 & 3.2 & $\mathrm{U}$ & 51.7 & 41.5 & 30.1 & $\mathrm{R}$ & & & \\
\hline HD 193901 & 3.8 & 2.2 & $\mathrm{U}, \mathrm{H}$ & 32.4 & 26.5 & 18.9 & $\mathrm{R}$ & & & \\
\hline HD 194598 & 5.7 & 2.6 & $\mathrm{U}$ & 45.6 & 38.2 & 27.6 & $\mathrm{R}$ & & & \\
\hline HD 199289 & 8.4 & 4.3 & $\mathrm{U}, \mathrm{H}$ & 52.0 & 44.3 & 33.1 & $\mathrm{R}$ & 2.4 & 2.2 & $\mathrm{U}, \mathrm{H}$ \\
\hline HD 205650 & 5.7 & 3.1 & $\mathrm{U}$ & 42.2 & 32.4 & 24.4 & $\mathrm{R}$ & & & \\
\hline HD 219617 & 2.1 & & $\mathrm{U}$ & 30.3 & 26.9 & 17.9 & $\mathrm{R}$ & & & \\
\hline HD 222766 & 9.9 & 6.6 & $\mathrm{U}$ & 42.9 & 37.2 & 25.0 & $\mathrm{R}$ & & & \\
\hline HD 230409 & 4.7 & & $\mathrm{~F}$ & 27.0 & 23.0 & 18.0 & $\mathrm{R}$ & & & \\
\hline HD 233511 & & & & 33.2 & 30.1 & 18.3 & $\mathrm{R}$ & & & \\
\hline HD 237822 & 20.9 & 12.8 & $\mathrm{~F}$ & & & & & & & \\
\hline HD 241253 & 7.5 & 3.8 & $\mathrm{U}$ & 52.4 & 41.3 & 33.3 & $\mathrm{R}, \mathrm{E}$ & & & \\
\hline HD 250792A & & & & 29.7 & 22.9 & & $\mathrm{R}$ & & & \\
\hline HD 284248 & 2.8 & 1.7 & $\mathrm{U}$ & 30.9 & 26.3 & 18.1 & $\mathrm{R}$ & & & \\
\hline
\end{tabular}


P. E. Nissen et al.: Carbon and oxygen abundances in stellar populations

Table 4. Atmospheric parameters and abundances for the HARPS-FEROS sample of disk stars.

\begin{tabular}{|c|c|c|c|c|c|c|c|c|c|c|c|}
\hline ID & $\begin{array}{l}T_{\text {eff }} \\
(\mathrm{K})\end{array}$ & $\log g$ & {$[\mathrm{Fe} / \mathrm{H}]$} & $\begin{array}{c}\begin{array}{c}\xi_{\text {turb }} \\
\mathrm{km} \mathrm{s}^{-1}\end{array} \\
\end{array}$ & $\begin{array}{r}{[\mathrm{C} / \mathrm{H}]} \\
\mathrm{LTE} \\
\end{array}$ & $\begin{array}{r}{[\mathrm{C} / \mathrm{H}]} \\
\text { non-LTE }\end{array}$ & $\begin{array}{r}{[\mathrm{O} / \mathrm{H}]_{7774}} \\
\mathrm{LTE} \\
\end{array}$ & $\begin{array}{c}{[\mathrm{O} / \mathrm{H}]_{7774}} \\
\text { non-LTE }\end{array}$ & {$[\mathrm{O} / \mathrm{H}]_{6300}$} & Pop. ${ }^{a}$ & $N_{\text {planet }^{b}}^{b}$ \\
\hline HD 1237 & 5507 & 4.56 & 0.14 & 1.25 & 0.09 & 0.09 & 0.09 & 0.16 & 0.07 & $\mathrm{D}$ & 0 \\
\hline HD 4208 & 5688 & 4.53 & -0.28 & 1.02 & -0.22 & -0.22 & -0.23 & -0.17 & & D & 1 \\
\hline HD 4307 & 5828 & 4.02 & -0.22 & 1.23 & -0.24 & -0.25 & -0.09 & -0.15 & -0.11 & $\mathrm{D}$ & 0 \\
\hline HD 4308 & 5705 & 4.36 & -0.35 & 1.00 & -0.19 & -0.19 & -0.04 & -0.02 & & $\mathrm{C}$ & 1 \\
\hline HD 14374 & 5466 & 4.58 & -0.03 & 0.84 & -0.07 & -0.07 & -0.08 & 0.01 & -0.02 & $\mathrm{D}$ & 0 \\
\hline HD 16141 & 5722 & 4.10 & 0.16 & 1.13 & 0.09 & 0.09 & 0.18 & 0.12 & & $\mathrm{D}$ & 1 \\
\hline HD 20782 & 5784 & 4.35 & -0.06 & 1.02 & -0.08 & -0.08 & -0.04 & -0.05 & -0.02 & $\mathrm{D}$ & 1 \\
\hline HD 23079 & 5988 & 4.38 & -0.14 & 1.15 & -0.18 & -0.18 & -0.13 & -0.17 & -0.09 & $\mathrm{D}$ & 1 \\
\hline HD 28185 & 5640 & 4.35 & 0.21 & 0.94 & 0.22 & 0.22 & 0.14 & 0.14 & 0.18 & $\mathrm{D}$ & 1 \\
\hline HD 30177 & 5550 & 4.39 & 0.50 & 0.92 & 0.39 & 0.39 & 0.33 & 0.34 & & $\mathrm{D}$ & 1 \\
\hline HD 30306 & 5544 & 4.38 & 0.22 & 0.90 & 0.16 & 0.16 & 0.16 & 0.19 & 0.10 & $\mathrm{D}$ & 0 \\
\hline HD 40397 & 5474 & 4.38 & -0.12 & 0.96 & 0.05 & 0.05 & 0.11 & 0.16 & 0.05 & $\mathrm{C}$ & 0 \\
\hline HD 52265 & 6129 & 4.32 & 0.26 & 1.27 & 0.15 & 0.14 & 0.18 & 0.04 & & $\mathrm{D}$ & 2 \\
\hline HD 65216 & 5658 & 4.52 & -0.16 & 0.98 & -0.20 & -0.19 & -0.12 & -0.07 & -0.08 & $\mathrm{D}$ & 2 \\
\hline HD 65907 & 5998 & 4.41 & -0.33 & 1.08 & -0.15 & -0.16 & 0.00 & -0.05 & 0.03 & $\mathrm{C}$ & 0 \\
\hline HD 69830 & 5460 & 4.55 & -0.03 & 0.63 & -0.04 & -0.04 & -0.12 & -0.03 & 0.00 & $\mathrm{D}$ & 3 \\
\hline HD 73256 & 5453 & 4.46 & 0.31 & 1.13 & 0.29 & 0.29 & 0.24 & 0.30 & 0.17 & $\mathrm{D}$ & 1 \\
\hline HD 75289 & 6121 & 4.34 & 0.28 & 1.32 & 0.11 & 0.11 & 0.16 & 0.03 & 0.03 & $\mathrm{D}$ & 1 \\
\hline HD 77110 & 5738 & 4.39 & -0.51 & 0.87 & -0.40 & -0.40 & -0.17 & -0.14 & -0.07 & $\mathrm{C}$ & 0 \\
\hline HD 78538 & 5790 & 4.50 & -0.04 & 1.06 & -0.17 & -0.17 & -0.08 & -0.07 & -0.08 & $\mathrm{D}$ & 0 \\
\hline HD 82943 & 5958 & 4.38 & 0.26 & 1.21 & 0.19 & 0.19 & 0.22 & 0.14 & 0.13 & $\mathrm{D}$ & 3 \\
\hline HD 89454 & 5699 & 4.53 & 0.19 & 0.94 & 0.06 & 0.06 & 0.12 & 0.14 & 0.04 & $\mathrm{D}$ & 0 \\
\hline HD 92788 & 5691 & 4.35 & 0.30 & 1.05 & 0.26 & 0.26 & 0.25 & 0.23 & 0.17 & $\mathrm{D}$ & 2 \\
\hline HD 94151 & 5621 & 4.50 & 0.09 & 0.89 & 0.06 & 0.06 & 0.03 & 0.07 & 0.00 & $\mathrm{D}$ & 0 \\
\hline HD 96423 & 5689 & 4.39 & 0.14 & 0.96 & 0.08 & 0.08 & 0.11 & 0.11 & 0.08 & $\mathrm{D}$ & 0 \\
\hline HD 102365 & 5690 & 4.46 & -0.31 & 0.91 & -0.19 & -0.19 & -0.21 & -0.17 & -0.09 & $\mathrm{D}$ & 1 \\
\hline HD 108147 & 6218 & 4.39 & 0.17 & 1.35 & 0.05 & 0.05 & 0.14 & 0.00 & -0.05 & $\mathrm{D}$ & 1 \\
\hline HD 110668 & 5808 & 4.39 & 0.17 & 1.08 & 0.18 & 0.18 & 0.20 & 0.16 & & $\mathrm{D}$ & 0 \\
\hline HD 111232 & 5543 & 4.43 & -0.48 & 0.90 & -0.20 & -0.20 & -0.07 & -0.01 & & $\mathrm{C}$ & 1 \\
\hline HD 114613 & 5700 & 3.90 & 0.14 & 1.33 & 0.11 & 0.10 & 0.16 & 0.07 & 0.07 & $\mathrm{D}$ & 1 \\
\hline HD 114729 & 5790 & 4.07 & -0.28 & 1.27 & -0.21 & -0.22 & -0.07 & -0.12 & -0.07 & $\mathrm{D}$ & 1 \\
\hline HD 114853 & 5744 & 4.46 & -0.23 & 0.97 & -0.25 & -0.25 & -0.22 & -0.19 & & $\mathrm{D}$ & 0 \\
\hline HD 115617 & 5534 & 4.41 & -0.01 & 0.91 & -0.03 & -0.03 & -0.07 & -0.01 & 0.01 & $\mathrm{D}$ & 3 \\
\hline HD 117207 & 5632 & 4.34 & 0.26 & 0.97 & 0.20 & 0.20 & 0.18 & 0.19 & & $\mathrm{D}$ & 1 \\
\hline HD 117618 & 5962 & 4.32 & 0.01 & 1.18 & 0.01 & 0.01 & 0.04 & -0.03 & -0.06 & $\mathrm{D}$ & 2 \\
\hline HD 125184 & 5610 & 4.07 & 0.33 & 1.14 & 0.27 & 0.26 & 0.27 & 0.23 & 0.09 & $\mathrm{D}$ & 0 \\
\hline HD 125612 & 5872 & 4.45 & 0.28 & 1.13 & 0.10 & 0.10 & 0.17 & 0.12 & 0.18 & $\mathrm{D}$ & 3 \\
\hline HD 126525 & 5650 & 4.41 & -0.10 & 0.92 & -0.06 & -0.06 & -0.09 & -0.06 & 0.00 & $\mathrm{D}$ & 1 \\
\hline HD 128674 & 5600 & 4.48 & -0.36 & 0.82 & -0.32 & -0.32 & -0.30 & -0.22 & -0.23 & $\mathrm{D}$ & 0 \\
\hline HD 134664 & 5805 & 4.39 & 0.05 & 1.11 & -0.01 & -0.01 & 0.02 & 0.00 & 0.00 & $\mathrm{D}$ & 0 \\
\hline HD 134987 & 5710 & 4.28 & 0.31 & 1.08 & 0.30 & 0.30 & 0.27 & 0.23 & 0.16 & $\mathrm{D}$ & 2 \\
\hline HD 136352 & 5728 & 4.36 & -0.39 & 1.05 & -0.18 & -0.18 & -0.08 & -0.07 & 0.00 & $\mathrm{C}$ & 3 \\
\hline HD 140901 & 5582 & 4.47 & 0.12 & 0.95 & 0.08 & 0.08 & 0.08 & 0.12 & -0.03 & $\mathrm{D}$ & 0 \\
\hline HD 145666 & 5918 & 4.47 & -0.04 & 1.09 & -0.10 & -0.10 & -0.01 & -0.03 & -0.06 & $\mathrm{D}$ & 0 \\
\hline HD 146233 & 5820 & 4.47 & 0.05 & 1.01 & -0.02 & -0.02 & -0.01 & -0.01 & 0.03 & $\mathrm{D}$ & 0 \\
\hline HD 157347 & 5688 & 4.41 & 0.03 & 0.96 & -0.03 & -0.03 & 0.00 & 0.01 & 0.04 & $\mathrm{D}$ & 0 \\
\hline HD 160691 & 5737 & 4.23 & 0.32 & 1.11 & 0.28 & 0.28 & 0.28 & 0.22 & 0.21 & $\mathrm{D}$ & 4 \\
\hline HD 168443 & 5522 & 4.06 & 0.07 & 1.02 & 0.17 & 0.16 & 0.23 & 0.22 & 0.14 & $\mathrm{C}$ & 2 \\
\hline HD 169830 & 6319 & 4.08 & 0.15 & 1.58 & 0.11 & 0.10 & 0.28 & 0.01 & 0.11 & $\mathrm{D}$ & 2 \\
\hline HD 179949 & 6182 & 4.36 & 0.20 & 1.34 & 0.12 & 0.12 & 0.21 & 0.06 & & $\mathrm{D}$ & 1 \\
\hline HD 183263 & 5889 & 4.29 & 0.29 & 1.23 & 0.25 & 0.25 & 0.22 & 0.14 & & $\mathrm{D}$ & 2 \\
\hline HD 190248 & 5572 & 4.31 & 0.44 & 0.94 & 0.38 & 0.38 & 0.34 & 0.34 & 0.22 & $\mathrm{D}$ & 0 \\
\hline HD 196050 & 5862 & 4.22 & 0.26 & 1.16 & 0.24 & 0.23 & 0.23 & 0.14 & 0.14 & $\mathrm{D}$ & 1 \\
\hline HD 196761 & 5486 & 4.56 & -0.26 & 0.75 & -0.22 & -0.21 & -0.32 & -0.22 & & $\mathrm{D}$ & 0 \\
\hline
\end{tabular}

Notes. ${ }^{(a)}$ Population classification: C, thick-disk; D, thin-disk. ${ }^{(b)}$ Number of planets detected, (http: //exoplanets . org, April 2014). 
Table 4. continued.

\begin{tabular}{lccccrrrrrrr}
\hline \hline ID & $\begin{array}{l}T_{\text {eff }} \\
\text { (K) }\end{array}$ & $\log g$ & {$[\mathrm{Fe} / \mathrm{H}]$} & $\begin{array}{c}\xi_{\text {turb }} \\
\mathrm{km} \mathrm{s}^{-1}\end{array}$ & $\begin{array}{r}{[\mathrm{C} / \mathrm{H}]} \\
\mathrm{LTE}\end{array}$ & $\begin{array}{r}{[\mathrm{C} / \mathrm{H}]} \\
\text { non-LTE }\end{array}$ & $\begin{array}{r}{[\mathrm{O} / \mathrm{H}]_{7774}} \\
\text { LTE }\end{array}$ & $\begin{array}{r}{[\mathrm{O} / \mathrm{H}]_{7774}} \\
\text { non-LTE }\end{array}$ & {$[\mathrm{O} / \mathrm{H}]_{6300}$} & ${\text { Pop. }{ }^{a}}^{N_{\text {planet }^{b}}}$ \\
\hline HD 202206 & 5746 & 4.44 & 0.29 & 1.11 & 0.15 & 0.15 & 0.09 & 0.08 & 0.05 & $\mathrm{D}$ & 2 \\
HD 203608 & 6180 & 4.35 & -0.66 & 1.30 & -0.57 & -0.58 & -0.41 & -0.45 & -0.42 & $\mathrm{D}$ & 0 \\
HD 206172 & 5662 & 4.62 & -0.19 & 0.84 & -0.21 & -0.21 & -0.11 & -0.05 & & $\mathrm{D}$ & 0 \\
HD 207129 & 5941 & 4.42 & -0.02 & 1.06 & -0.11 & -0.12 & -0.06 & -0.09 & 0.00 & $\mathrm{D}$ & 0 \\
HD 210277 & 5479 & 4.34 & 0.24 & 0.84 & 0.28 & 0.28 & 0.26 & 0.29 & 0.17 & $\mathrm{C}$ & 1 \\
HD 212301 & 6162 & 4.35 & 0.16 & 1.35 & 0.11 & 0.10 & 0.17 & 0.03 & & $\mathrm{D}$ & 1 \\
HD 213240 & 5918 & 4.15 & 0.13 & 1.25 & 0.10 & 0.09 & 0.16 & 0.05 & 0.04 & $\mathrm{D}$ & 1 \\
HD 216435 & 5942 & 4.08 & 0.25 & 1.36 & 0.20 & 0.19 & 0.26 & 0.11 & 0.07 & $\mathrm{D}$ & 1 \\
HD 216437 & 5802 & 4.17 & 0.28 & 1.14 & 0.24 & 0.24 & 0.25 & 0.17 & 0.17 & $\mathrm{D}$ & 1 \\
HD 216770 & 5424 & 4.53 & 0.37 & 0.79 & 0.29 & 0.30 & 0.18 & 0.26 & 0.17 & $\mathrm{C}$ & 1 \\
HD 216777 & 5656 & 4.47 & -0.38 & 0.89 & -0.35 & -0.35 & -0.28 & -0.22 & & $\mathrm{D}$ & 0 \\
HD 222669 & 5908 & 4.48 & 0.08 & 1.01 & 0.00 & 0.00 & 0.02 & -0.01 & 0.02 & $\mathrm{D}$ & 0 \\
\hline
\end{tabular}

Table 5. Atmospheric parameters and abundances for the UVES-FIES sample of halo and thick-disk stars.

\begin{tabular}{|c|c|c|c|c|c|c|c|c|c|c|c|}
\hline ID & $\begin{array}{l}T_{\text {eff }} \\
(\mathrm{K})\end{array}$ & $\log g$ & {$[\mathrm{Fe} / \mathrm{H}]$} & $\begin{array}{c}\xi_{\text {turb }} \\
\mathrm{km} \mathrm{s}^{-1} \\
\end{array}$ & $\begin{array}{c}{[\mathrm{C} / \mathrm{H}]} \\
\mathrm{LTE}\end{array}$ & $\begin{array}{c}{[\mathrm{C} / \mathrm{H}]} \\
\text { non-LTE }\end{array}$ & $\begin{array}{c}{[\mathrm{O} / \mathrm{H}]_{7774}} \\
\mathrm{LTE}\end{array}$ & $\begin{array}{c}{[\mathrm{O} / \mathrm{H}]_{7774}} \\
\text { non-LTE }\end{array}$ & {$[\mathrm{O} / \mathrm{H}]_{6300}$} & Pop. ${ }^{a}$ & Bin. $^{b}$ \\
\hline BD-213420 & 5909 & 4.30 & -1.14 & 1.12 & -0.91 & -0.91 & -0.50 & -0.49 & -0.52 & $\mathrm{C}$ & \\
\hline CD-33 3337 & 6112 & 3.86 & -1.37 & 1.56 & -1.19 & -1.21 & -0.81 & -0.86 & & $\mathrm{C}$ & \\
\hline CD-43 6810 & 6059 & 4.32 & -0.44 & 1.24 & -0.24 & -0.24 & 0.00 & -0.08 & 0.00 & A & \\
\hline CD-45 3283 & 5685 & 4.61 & -0.93 & 0.95 & -1.02 & -1.02 & -0.60 & -0.50 & & $\mathrm{~B}$ & \\
\hline CD-51 4628 & 6296 & 4.29 & -1.32 & 1.31 & -1.40 & -1.41 & -0.98 & -0.98 & & B & \\
\hline CD-57 1633 & 5981 & 4.29 & -0.91 & 1.08 & -0.96 & -0.97 & -0.64 & -0.62 & & B & \\
\hline CD-61 0282 & 5869 & 4.34 & -1.25 & 1.19 & -1.29 & -1.29 & -0.75 & -0.70 & & $\mathrm{~B}$ & \\
\hline G05-19 & 5770 & 4.28 & -1.19 & 1.17 & -1.25 & -1.25 & -0.78 & -0.71 & & $\mathrm{~B}$ & \\
\hline G05-36 & 6139 & 4.22 & -1.25 & 1.29 & -1.12 & -1.12 & -0.67 & -0.69 & & A & \\
\hline G05-40 & 5892 & 4.20 & -0.83 & 1.12 & -0.62 & -0.63 & -0.27 & -0.29 & & $\mathrm{~A}$ & \\
\hline G15-23 & 5373 & 4.63 & -1.12 & 0.90 & -0.87 & -0.87 & -0.52 & -0.38 & & A & \\
\hline G18-28 & 5443 & 4.49 & -0.85 & 0.88 & -0.59 & -0.59 & -0.31 & -0.20 & & A & SB1 \\
\hline G18-39 & 6175 & 4.21 & -1.41 & 1.37 & -1.32 & -1.33 & -0.88 & -0.88 & & A & \\
\hline G20-15 & 6162 & 4.32 & -1.50 & 1.50 & -1.44 & -1.45 & -1.07 & -1.04 & & B & \\
\hline G21-22 & 6021 & 4.27 & -1.10 & 1.30 & -1.19 & -1.19 & & & & B & \\
\hline G24-13 & 5764 & 4.38 & -0.73 & 0.86 & -0.59 & -0.59 & -0.14 & -0.12 & & A & \\
\hline G31-55 & 5731 & 4.35 & -1.12 & 1.26 & -0.92 & -0.92 & -0.58 & -0.51 & & A & \\
\hline G46-31 & 6017 & 4.29 & -0.83 & 1.30 & -0.76 & -0.77 & -0.50 & -0.51 & -0.41 & B & SB1 \\
\hline G49-19 & 5863 & 4.32 & -0.55 & 1.12 & -0.37 & -0.37 & & & & A & SB1 \\
\hline G53-41 & 5975 & 4.29 & -1.21 & 1.20 & -1.38 & -1.39 & -1.06 & -1.00 & & B & \\
\hline G56-30 & 5935 & 4.29 & -0.90 & 1.22 & -1.02 & -1.02 & & & & B & \\
\hline G56-36 & 6067 & 4.33 & -0.94 & 1.33 & -0.87 & -0.88 & -0.51 & -0.52 & & B & \\
\hline G57-07 & 5755 & 4.33 & -0.48 & 0.99 & -0.33 & -0.33 & & & & A & \\
\hline G63-26 & 6175 & 4.17 & -1.58 & 1.65 & -1.36 & -1.37 & & & & A & \\
\hline G66-22 & 5297 & 4.46 & -0.88 & 0.78 & -0.98 & -0.98 & -0.59 & -0.46 & & B & \\
\hline G74-32 & 5864 & 4.41 & -0.74 & 1.04 & -0.54 & -0.54 & & & & A & \\
\hline G75-31 & 6135 & 4.02 & -1.04 & 1.28 & -1.17 & -1.18 & -0.72 & -0.75 & & B & \\
\hline G81-02 & 5967 & 4.24 & -0.69 & 1.21 & -0.57 & -0.57 & & & & A & \\
\hline G82-05 & 5338 & 4.51 & -0.78 & 0.80 & -0.69 & -0.69 & -0.51 & -0.38 & & B & \\
\hline G85-13 & 5709 & 4.46 & -0.60 & 0.87 & -0.46 & -0.46 & -0.15 & -0.11 & & A & \\
\hline G87-13 & 6217 & 4.11 & -1.10 & 1.42 & -1.34 & -1.35 & & & & B & \\
\hline G96-20 & 6445 & 4.46 & -0.90 & 1.42 & -0.62 & -0.63 & & & & A & \\
\hline G98-53 & 5954 & 4.26 & -0.89 & 1.20 & -0.83 & -0.84 & & & & B & \\
\hline G99-21 & 5559 & 4.46 & -0.68 & 0.79 & -0.41 & -0.41 & -0.16 & -0.09 & & A & \\
\hline G112-43 & 6209 & 4.02 & -1.27 & 1.17 & -1.23 & -1.24 & -0.88 & -0.91 & & B & \\
\hline G112-44 & 5936 & 4.28 & -1.31 & 1.10 & -1.21 & -1.21 & & & & B & \\
\hline
\end{tabular}

Notes. ${ }^{(a)}$ Population classification: A, high-alpha halo; B, low-alpha halo; C, thick-disk. ${ }^{(b)}$ Note on binarity: SB1, single-lined spectroscopic binary according to the SIMBAD database; D, double star according to the HIPPARCOS and TYCHO catalogues (Perryman et al. 1997). 
P. E. Nissen et al.: Carbon and oxygen abundances in stellar populations

Table 5. continued.

\begin{tabular}{|c|c|c|c|c|c|c|c|c|c|c|c|}
\hline ID & $\begin{array}{l}T_{\text {eff }} \\
(\mathrm{K}) \\
\end{array}$ & $\log g$ & {$[\mathrm{Fe} / \mathrm{H}]$} & $\begin{array}{c}\xi_{\text {turb }} \\
\mathrm{km} \mathrm{s}^{-1} \\
\end{array}$ & $\begin{array}{c}{[\mathrm{C} / \mathrm{H}]} \\
\mathrm{LTE}\end{array}$ & $\begin{array}{c}{[\mathrm{C} / \mathrm{H}]} \\
\text { non-LTE }\end{array}$ & $\begin{array}{c}{[\mathrm{O} / \mathrm{H}]_{7774}} \\
\mathrm{LTE}\end{array}$ & $\begin{array}{c}{[\mathrm{O} / \mathrm{H}]_{7774}} \\
\text { non-LTE }\end{array}$ & {$[\mathrm{O} / \mathrm{H}]_{6300}$} & Pop. ${ }^{a}$ & Bin. $^{b}$ \\
\hline G114-42 & 5721 & 4.40 & -1.12 & 1.19 & -1.10 & -1.11 & & & & B & \\
\hline G119-64 & 6333 & 4.14 & -1.50 & 1.40 & -1.53 & -1.54 & -1.08 & -1.09 & & B & \\
\hline G121-12 & 6041 & 4.25 & -0.94 & 1.26 & -1.09 & -1.10 & & & & B & \\
\hline G127-26 & 5886 & 4.20 & -0.53 & 1.11 & -0.37 & -0.38 & & & & A & \\
\hline G150-40 & 6080 & 4.11 & -0.82 & 1.31 & -1.07 & -1.08 & -0.63 & -0.66 & & B & \\
\hline G159-50 & 5713 & 4.44 & -0.94 & 1.03 & -0.70 & -0.70 & -0.48 & -0.41 & & A & \\
\hline G161-73 & 6108 & 3.99 & -1.01 & 1.26 & -1.18 & -1.19 & & & & B & \\
\hline G170-56 & 6112 & 4.11 & -0.94 & 1.39 & -0.96 & -0.97 & -0.62 & -0.65 & & B & \\
\hline G176-53 & 5615 & 4.52 & -1.36 & 0.90 & & & -0.85 & -0.73 & & B & \\
\hline G180-24 & 6137 & 4.20 & -1.41 & 1.45 & -1.17 & -1.18 & -0.82 & -0.82 & & A & \\
\hline G187-18 & 5691 & 4.46 & -0.68 & 1.05 & -0.44 & -0.44 & & & & A & \\
\hline G188-22 & 6116 & 4.20 & -1.33 & 1.42 & -1.18 & -1.19 & -0.68 & -0.70 & & A & \\
\hline HD 3567 & 6180 & 4.01 & -1.17 & 1.40 & -1.26 & -1.28 & -0.83 & -0.86 & & B & \\
\hline HD 17820 & 5873 & 4.28 & -0.68 & 1.27 & -0.46 & -0.47 & -0.18 & -0.20 & -0.21 & $\mathrm{C}$ & \\
\hline HD 22879 & 5859 & 4.29 & -0.86 & 1.20 & -0.64 & -0.64 & -0.29 & -0.29 & & $\mathrm{C}$ & \\
\hline HD 25704 & 5974 & 4.30 & -0.86 & 1.33 & -0.67 & -0.68 & -0.44 & -0.44 & -0.40 & $\mathrm{C}$ & $\mathrm{D}$ \\
\hline HD 51754 & 5857 & 4.35 & -0.58 & 1.30 & -0.39 & -0.39 & -0.16 & -0.17 & -0.19 & A & \\
\hline HD 59392 & 6137 & 3.88 & -1.62 & 1.73 & -1.61 & -1.62 & -1.12 & -1.12 & & $\mathrm{~B}$ & \\
\hline HD 76932 & 5977 & 4.17 & -0.87 & 1.30 & -0.73 & -0.73 & -0.37 & -0.40 & -0.30 & $\mathrm{C}$ & \\
\hline HD 97320 & 6136 & 4.20 & -1.18 & 1.46 & -0.96 & -0.97 & -0.66 & -0.68 & & $\mathrm{C}$ & \\
\hline HD 103723 & 6050 & 4.20 & -0.81 & 1.11 & -0.86 & -0.86 & -0.54 & -0.56 & & B & \\
\hline HD 105004 & 5852 & 4.35 & -0.83 & 1.09 & -0.73 & -0.73 & -0.56 & -0.52 & & $\mathrm{~B}$ & \\
\hline HD 106516 & 6327 & 4.43 & -0.69 & 1.18 & -0.51 & -0.51 & -0.11 & -0.22 & & $\mathrm{C}$ & SB1 \\
\hline HD 111980 & 5878 & 3.98 & -1.09 & 1.39 & -0.92 & -0.93 & -0.43 & -0.46 & -0.53 & $\mathrm{~A}$ & SB1 \\
\hline HD 113679 & 5761 & 4.05 & -0.66 & 1.37 & -0.53 & -0.53 & -0.17 & -0.19 & -0.24 & $\mathrm{~A}$ & \\
\hline HD 114762A & 5956 & 4.24 & -0.72 & 1.37 & -0.53 & -0.54 & -0.28 & -0.31 & & $\mathrm{C}$ & SB1 \\
\hline HD 120559 & 5486 & 4.58 & -0.91 & 1.05 & -0.61 & -0.60 & -0.39 & -0.26 & -0.25 & $\mathrm{C}$ & \\
\hline HD 121004 & 5755 & 4.43 & -0.71 & 1.16 & -0.52 & -0.52 & -0.26 & -0.22 & -0.20 & A & \\
\hline HD 126681 & 5594 & 4.50 & -1.20 & 1.08 & -0.98 & -0.98 & -0.61 & -0.50 & & $\mathrm{C}$ & \\
\hline HD 132475 & 5750 & 3.77 & -1.51 & 1.37 & -1.35 & -1.36 & -0.82 & -0.79 & & A & \\
\hline HD 148816 & 5923 & 4.17 & -0.74 & 1.33 & -0.53 & -0.53 & -0.24 & -0.28 & & A & \\
\hline HD 159482 & 5829 & 4.37 & -0.74 & 1.21 & -0.54 & -0.55 & -0.20 & -0.19 & & A & $\mathrm{D}$ \\
\hline HD 160693 & 5809 & 4.35 & -0.48 & 1.02 & -0.37 & -0.37 & -0.09 & -0.10 & & A & \\
\hline HD 163810 & 5592 & 4.61 & -1.22 & 1.17 & -1.17 & -1.17 & -0.79 & -0.67 & & B & $\mathrm{D}$ \\
\hline HD 175179 & 5804 & 4.40 & -0.66 & 1.08 & -0.42 & -0.42 & -0.09 & -0.09 & -0.14 & $\mathrm{C}$ & \\
\hline HD 179626 & 5957 & 4.16 & -1.06 & 1.47 & -0.87 & -0.87 & -0.47 & -0.48 & & A & \\
\hline HD 189558 & 5707 & 3.83 & -1.14 & 1.29 & -1.05 & -1.06 & -0.51 & -0.51 & & $\mathrm{C}$ & \\
\hline HD 193901 & 5745 & 4.42 & -1.11 & 1.12 & -1.05 & -1.05 & -0.71 & -0.63 & & B & \\
\hline HD 194598 & 6053 & 4.33 & -1.11 & 1.30 & -1.13 & -1.14 & -0.72 & -0.70 & & B & \\
\hline HD 199289 & 5915 & 4.30 & -1.05 & 1.21 & -0.87 & -0.88 & -0.50 & -0.49 & -0.47 & $\mathrm{C}$ & \\
\hline HD 205650 & 5793 & 4.35 & -1.19 & 1.17 & -0.94 & -0.94 & -0.60 & -0.54 & & $\mathrm{C}$ & \\
\hline HD 219617 & 5983 & 4.28 & -1.46 & 1.42 & -1.53 & -1.53 & -0.95 & -0.91 & & B & $\mathrm{D}$ \\
\hline HD 222766 & 5423 & 4.38 & -0.70 & 0.75 & -0.41 & -0.41 & -0.16 & -0.07 & & A & \\
\hline HD 230409 & 5386 & 4.61 & -0.87 & 1.01 & -0.71 & -0.70 & -0.40 & -0.27 & & A & \\
\hline HD 233511 & 6125 & 4.21 & -1.58 & 1.20 & & & -1.02 & -0.99 & & A & \\
\hline HD 237822 & 5675 & 4.41 & -0.47 & 0.99 & -0.23 & -0.23 & & & & A & \\
\hline HD 241253 & 5940 & 4.34 & -1.11 & 1.17 & -0.92 & -0.93 & -0.52 & -0.51 & & $\mathrm{C}$ & \\
\hline HD 250792A & 5572 & 4.50 & -1.03 & 0.98 & & & -0.62 & -0.50 & & $\mathrm{~B}$ & $\mathrm{D}$ \\
\hline HD 284248 & 6271 & 4.21 & -1.59 & 1.51 & -1.52 & -1.53 & -1.17 & -1.15 & & $\mathrm{~B}$ & \\
\hline
\end{tabular}

